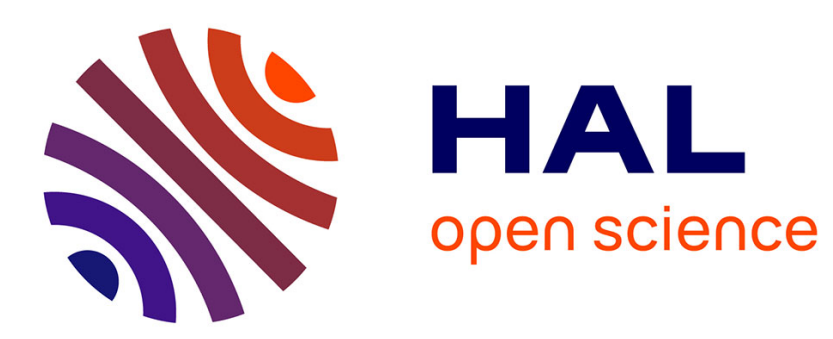

\title{
La contribution des TIC et du présentiel à l'efficacité de la coordination des équipes projets de NPD distribuées
}

Sébastien Brion, Caroline Mothe, Céline Perea

\section{To cite this version:}

Sébastien Brion, Caroline Mothe, Céline Perea. La contribution des TIC et du présentiel à l'efficacité de la coordination des équipes projets de NPD distribuées. Systèmes d'Information et Management, 2013, 18 (4), pp.43-75. 10.3917/sim.134.0043 . hal-01300931

\section{HAL Id: hal-01300931 \\ https://hal.univ-grenoble-alpes.fr/hal-01300931}

Submitted on 11 Apr 2016

HAL is a multi-disciplinary open access archive for the deposit and dissemination of scientific research documents, whether they are published or not. The documents may come from teaching and research institutions in France or abroad, or from public or private research centers.
L'archive ouverte pluridisciplinaire HAL, est destinée au dépôt et à la diffusion de documents scientifiques de niveau recherche, publiés ou non, émanant des établissements d'enseignement et de recherche français ou étrangers, des laboratoires publics ou privés. 


\title{
La contribution des TIC et du présentiel à l'efficacité de la coordination des équipes projets de NPD distribuées
}

\author{
Sébastien BRION*, Caroline MOTHE*\& Céline PEREA**
}

* IREGE, Université de Savoie

** CERAG, Université Pierre Mendès France

\begin{abstract}
RÉSUMÉ
Une plus grande distribution géographique et temporelle caractérise depuis une vingtaine d'année les équipes de développement de nouveaux produits (NPD). Certains mettent en avant que cette distribution entrave la coordination de ces équipes, notamment parce qu'elle limite les occasions d'échanges et restreint le recours au présentiel, d'autres considèrent au contraire qu'une coordination par les TIC est tout aussi efficace. Bien que relativement ancien, ce débat n'a jamais fait l'objet de validation empirique sur des projets de NPD. Pour combler ce gap, nous étudions l'influence des médias tant technologiques que non technologiques sur la coordination selon le niveau de distribution des membres des équipes de NPD. Les résultats de la modélisation structurelle avec la méthode PLS indiquent que l'effet des différents médias sur la coordination varie selon le niveau de distribution spatio-temporelle. Ils remettent en question la pertinence des échanges en présentiel lorsque les équipes projets sont très distribuées, équipes pour lesquelles la coordination repose davantage sur l'utilisation fréquente des TIC (d'aide à la conception et support de l'organisation des tâches). Aussi cette recherche indique-t-elle que la richesse des supports n'explique pas, à elle seule, l'efficacité des médias utilisés. Nous contribuons aussi à la littérature qui tend à assimiler la distribution au type de média utilisé en montrant que les deux concepts sont différents.
\end{abstract}

Mots-clés : Coordination, Equipes distribuées, Projets de NPD, Présentiel, TIC 
In the last twenty years, new product development (NPD) teams have been characterized by increased spatial and temporal distribution. Some authors assert that this distribution binders the coordination of these teams, especially because it limits the opportunities for exchange as well as the use of face-to-face dialogue. Other authors consider that coordination through ICT is as effective as face-to-face communication. Although scholars have been debating this point for years, the issue has never been empirically validated in the case of NDP project teams. To fill this gap, we study the influence of technological and nontechnological media on coordination by taking into account the distribution level of NPD team members. Results of PLS modelling indicate that the effect of the various media on Coordination varies according to the level of spatial and temporal distribution. The results dispute the relevance of face-to-face exchanges when project teams are highly distributed, teams in which coordination is frequently based on the use of ICT (for design support and for task organization). Therefore, this study indicates that the richness of media does not alone explain the media's efficiency. We also contribute to the literature which tends to equate team distribution to the media type by showing that the two concepts are different.

Keywords: Coordination, Distributed teams, NPD projects, Face-to-face, ICT 


\section{INTRODUCTION}

Les équipes projets de développement de produits nouveaux (NPD, New Product Development) convertissent un concept en un produit final en un temps donné (Tidd et Bodley, 2002). Le processus de NPD succède à la phase de recherche amont et s'étend jusqu'au développement de la première série du produit nouveau (Clark et al. 1987 ; Moenaert et al., 2000). Depuis une vingtaine d'années, on assiste à une plus grande distribution géographique des équipes projets en charge de ce processus (Harvey et Griffith, 2007 ; McDonough et al. 2001). Cette distribution géographique s'accompagne souvent d'une distribution temporelle des équipes sur différents fuseaux horaires (Gupta et al. 2009).

D'un côté, la littérature sur les projets de NPD présente la distribution des équipes comme une source d'avantage concurrentiel dans la mesure où celle-ci permet l'accès à des savoir-faire et connaissances spécifiques localisés dans le monde entier (Salomo et al. 2010 ; Gassmann et von Zedtwitz, 2003). L'étendue géographique de ces équipes offre ainsi un accès à une large base de connaissances (Boutellier et al. 1998 ; Sole et Edmondson, 2002), de technologies et d'experts (Eppinger et Chitkara, 2006). De l'autre, certains travaux mettent en avant que la distribution des équipes peut entraver la coordination de leurs tâches au cours des projets de NPD (Hoegl et al. 2004 ; O'Leary et Cummings, 2007), neutralisant ainsi en partie les avantages susmentionnés. Notre recherche s'insère dans ce dilemme dans la littérature lié à la coordination des projets de NPD, no- tamment dans un contexte distribué. Afin de coordonner les tâches des équipes projets distribuées, c'est à dire d'identifier, d'assembler, d'organiser et d'assigner les tâches aux membres de l'équipe dans le but d'atteindre un objectif commun (Malone et Crowston, 1990), différents types de médias sont utilisés. La distribution spatio-temporelle des équipes ayant pour conséquence de désincarner le travail (Child et McGrath, 2001 ; Daassi et Favier, 2007) et de diminuer la fréquence des communications en présentiel (Grinter et al. 1990 ; Kirkman et Mathieu, 2005), un recours aux technologies de l'information et de la communication (TIC) est souvent nécessaire pour échanger à distance (Stone et Posey, 2008).

Là encore, une controverse importante existe dans la littérature : d'un côté, les échanges en présentiel sont vus comme facilitant la coordination des équipes projets et complémentaires des échanges réalisés par le biais des TIC (Salter et Gann, 2003). En effet, le présentiel favoriserait la socialisation et la coordination de ces équipes, et permet de consolider la confiance et l'engagement mutuel dans les grands projets d'innovation distribués (Orlikowski, 2002). De l'autre, l'usage des TIC pour coordonner les tâches a été perçu comme corrélé à ce niveau de distribution (Bierly et al. 2009 ; Hertel et al. 2004 ; Kim et Kim, 2009), incitant certains auteurs (e.g., Malhotra et al. 2001 ; Michaud, 2005) à indiquer que le succès des projets distribués ne dépend pas des modes de coordination en présentiel. D'autres auteurs (Griffith et Neale, 2001, 2003 ; Fiol et O'Connor, 2005) vont même jusqu'à assimiler le degré de distribution (ou de "virtuali- 
té ") des équipes aux médias utilisés, voire à confondre les deux notions. Si une forte corrélation entre ces notions peut exister, elles ne semblent toutefois pas substituables au regard des recherches antérieures. Nous partons donc du postulat qu'il est nécessaire de distinguer le niveau de distribution spatiale et temporelle des supports de communication utilisés.

Notre objectif est d'apporter une réponse empirique à cette controverse, qui reste essentiellement de nature théorique, en étudiant l'effet des médias (TIC et présentiel) sur la coordination des équipes de NPD - et ce selon le niveau de distribution spatio-temporelle des équipes. Notre problématique est donc la suivante: "Quels sont les médias les plus efficaces pour coordonner les équipes projets de NPD distribuées "?

Pour traiter cette question, nous exposons dans la première partie les enjeux de la coordination des tâches dans les équipes projets de NPD et les médias qui favorisent l'efficacité de cette coordination. Les travaux de recherche et typologies existantes nous conduisent à distinguer les médias technologiques (TIC pour organiser les tâches, pour la conception des produits et pour communiquer de façon synchrone) et non technologiques (échanges en présentiel). Dans la deuxième partie, nous croisons les approches théoriques sur l'usage des médias et la coordination des tâches dans les équipes distribuées pour examiner les médias utilisés par les équipes distribuées de NPD tout en analysant le rôle modérateur de la distribution spatio-temporelle des équipes.
Cette partie débouche sur notre modèle de recherche reposant sur six hypothèses principales. La troisième partie présente la démarche méthodologique, la quatrième développant les résultats empiriques. L'analyse des données recueillies par modélisation structurelle met en évidence les effets différenciés des médias utilisés pour coordonner les tâches dans les équipes de NPD selon leur niveau de distribution, résultats qui sont discutés dans la cinquième partie. Les limites de la recherche, ainsi que des perspectives de recherches futures, sont finalement exposées.

\section{LES MEDIAS POUR COORDONNER LES ÉQUIPES PROJETS DE NPD}

Les équipes projets de NPD rassemblent plusieurs métiers : R\&D, conception, marketing, méthodes, approvisionnement, qualité, etc. (Gerwin et Barrowman, 2002 ; Holland et al. 2000 ; Kim et Kim, 2009). Elles réalisent des tâches plus ou moins interdépendantes qu'il est donc nécessaire de coordonner. Le processus de coordination est défini par Malone et Crowston (1990, p. 361) comme "l'action d'organiser les interdépendances entre des activités accomplies pour atteindre un objectif $n^{1}$. Dans les projets de NPD, la coordination repose sur le travail collectif des membres des équipes projets (Brown et Eisenhardt, 1995, Olson et al. 1995), essentiellement par ajustements mutuels (Thompson, 1967), ainsi que sur la rationalisation du processus d'innovation - rationalisation entendue au sens de

\footnotetext{
${ }^{1}$ Malone et Crowston (1990, p. 361) : " the act of managing interdependencies between activities performed to achieve a goal ".
} 
planification et formalisation du travail (Brown et Eisenhardt, 1995). Les ajustements mutuels reposent sur les communications entre membres de l'équipe (Michaux, 2005). Ces échanges leur permettent de prendre connaissance de ce que chacun fait (Allen et Henn, 2006), de réduire le risque d'incompréhensions des flux d'informations (Brown et Eisenhardt, 1995), et de réaliser le travail commun.

La coordination des tâches dans les équipes projets de NPD est dite " efficace " lorsqu'elle repose sur la qualité de leur collaboration (Kahn et McDonough, 1997), de leur coopération (Moenaert et al. 2000) et de la résolution collective des problèmes qui surviennent au cours du projet (Banker et al. 2006). La coordination des projets de NPD est un processus difficile, car réalisé sous contrainte temporelle (Clark et al. 1987 ; Krishnan et Ulrich, 2001). La qualité de cette coordination est un facteur important de la fiabilité et de la vitesse de réalisation des projets de NPD (Brion, 2005).

Différents types de TIC sont utilisés par les équipes projets de NPD. Boutellier et al. (1998) en distinguent jusqu'à 17 pour des projets de développement logiciels. Barczak et al. (2008), étudiant des projets de NPD, les TIC permettent d'accompagner à la fois le processus de développement et la gestion des différents projets. Pavlou et al. (2010) proposent une typologie permettant de regrouper les TIC supports des projets de NPD en trois catégories : pour le management des ressources du projet (Project Resource Management Systems PRMS), pour les connaissances et la mémoire organisationnelle (Organizational Memory Systems - OMS) et pour la communication et la coopération ( $\mathrm{CO}$ operative Work Systems - CWS). Durmusoglu et Barczak (2011) distinguent quant à eux 11 supports comme les emails, les outils de partage de documents, de génération d'idées, de conception de produits, soulignant l'impact positif de ces TIC selon les phases des projets. Le tableau 1 en page suivante propose une comparaison des supports utilisés par les équipes projets de NPD.

Au cours des projets de NPD, les TIC pour organiser les tâches permettent de gérer l'allocation des ressources ainsi que la planification et la répartition du travail dans l'équipe (Pavlou et al. 2010). Ces technologies offrent la possibilité d'informer régulièrement l'équipe sur le déroulement du projet grâce à un accès en ligne aux informations (Durmusoglu et Barczak, 2011 ; Hameri et Nihtilä, 1997). Les logiciels de planification et de suivi des tâches (comme $M S$ Project ou PSN) permettent à l'équipe de partager une vision commune de l'enchaînement optimal des tâches grâce à des techniques d'ordonnancement (comme le PERT, CPM, GANTT) et de suivre en temps réel les modifications d'affectation de ressources opérées par les responsables de tâches (Kumar, 2005 ; Yakura, 2002).

Ces TIC permettent ainsi de formaliser l'organisation du travail tout en rassemblant et diffusant des informations sur les tâches (Yalcin et al. 2005). C'est donc un moyen de standardiser la gestion du processus dans le temps (Yakura, 2002).

Toutefois, l'influence de la standardisation du processus de NPD sur la performance du projet est parfois remise 
Tableau 1 : TIC supports des équipes projets de NPD

\begin{tabular}{|l|l|l|l|}
\hline \multicolumn{1}{|c|}{ Auteurs } & $\begin{array}{c}\text { TIC supports de } \\
\text { l'organisation des tâches }\end{array}$ & \multicolumn{1}{c|}{$\begin{array}{c}\text { TIC d'aide } \\
\text { à la conception }\end{array}$} & \multicolumn{1}{c|}{$\begin{array}{c}\text { TIC supports } \\
\text { de communication }\end{array}$} \\
\hline Boutellier et al. (1998) & $\begin{array}{l}\text { Systèmes de management } \\
\text { de projet } \\
\text { Calendrier en ligne }\end{array}$ & $\begin{array}{l}\text { CAO, Bases de données, } \\
\text { Ftp... }\end{array}$ & $\begin{array}{l}\text { Téléphone, e-mails, } \\
\text { LAN/WAN, vidéo-confé- } \\
\text { rence, Intranet, Forums... }\end{array}$ \\
\hline Nambisan (2003) & $\begin{array}{l}\text { TIC de gestion de projet } \\
\text { et de gestion de process } \\
\text { NPD }\end{array}$ & $\begin{array}{l}\text { SI de gestion des connais- } \\
\text { sances, Interface de desi- } \\
\text { gn virtuel, PDM... }\end{array}$ & $\begin{array}{l}\text { TIC de collaboration et de } \\
\text { communication }\end{array}$ \\
\hline Pavlou et El Sawi (2010) & $\begin{array}{l}\text { TIC de management des } \\
\text { ressources du projet } \\
\text { (PRMS) }\end{array}$ & $\begin{array}{l}\text { TIC de gestion des } \\
\text { connaissances et de la } \\
\text { mémoire de l'organisation } \\
\text { (OMS) }\end{array}$ & $\begin{array}{l}\text { TIC de communication et } \\
\text { (CWS) }\end{array}$ \\
\hline $\begin{array}{l}\text { Durmusoglion temps réel } \\
\text { (2011) }\end{array}$ & $\begin{array}{l}\text { Interface de gestion parta- } \\
\text { gée des projets }\end{array}$ & $\begin{array}{l}\text { CAO/IAO en interface } \\
\text { web, outils distants de de- } \\
\text { sign des produits }\end{array}$ & $\begin{array}{l}\text { Outils de communication, } \\
\text { e-mail, web-meeting }\end{array}$ \\
\hline
\end{tabular}

en cause. Certains travaux soulignent le risque de rigidités dans le projet lorsque le processus de NPD est trop formalisé. L'étude de 120 projets de Sethi et Iqbal (2008) soutient quantitativement que la standardisation du processus de NPD peut diminuer les capacités des équipes à faire face aux imprévus et nuire à leur flexibilité. Pourtant, selon Kumar (2005), l'usage des technologies pour organiser les tâches permettrait de suivre le processus de coordination et favoriserait le respect des coûts et délais des projets de NPD. La relation entre l'usage des technologies pour organiser les tâches et l'efficacité de la coordination n'est donc pas évidente. Au regard de la littérature, nous faisons l'hypothèse suivante :

Hypotbèse $n^{\circ} 1$ : "L'utilisation des TIC pour organiser les tâches dans les équipes projets de NPD influence positivement l'efficacité de la coordination"

Les TIC d'aide à la conception (CAO, Conception Assistée par Ordinateur) accompagnent la conception d'un produit nouveau et/ou la modification d'un produit existant (Tan et Vonderembse, 2006). Ces outils permettent d'élaborer des dessins techniques, de fixer les spécifications d'un produit nouveau et ses méthodes d'industrialisation (Tan et Vonderembse, 2006) et de simuler l'ingénierie du produit (Baba et Nobeoka, 1998 ; Sun, 2000). Leur principale richesse provient de leurs capacités de simulation qui facilitent l'identification d'alternatives techniques (Tan et Vonderembse, 2006). En outre, elles offrent la possibilité de rassembler et stocker des documentations relatives au produit (Sun, 2000), et de capitaliser des informations et connaissances. Par ailleurs, elles procurent un langage commun aux membres de l'équipe (Tan et Vonderembse, 2006) et regroupent des données relatives aux différents métiers qui les composent (Koufteros et al. 2002).

L'ensemble de ces fonctionnalités, aujourd'hui intégré dans des technologies de type PLM (Product Lifecycle Management), permet d'anticiper les erreurs 
et les retours en arrière pendant le processus de développement (Merminod et al. 2009 ; Merminod et Rowe, 2012). L'étude longitudinale d'un projet de NPD (Merminod et al. 2009) indique que l'usage de PLM favorise la fiabilité et la productivité au cours du projet. À travers l'étude de 175 utilisateurs de la technologie CAO, Tan et Vonderembse (2006) montrent que l'usage de l'ensemble des fonctionnalités des outils $\mathrm{CAO}$ agit positivement sur la performance des projets de NPD. Ce lien positif a également été démontré à partir des données de l'IMSS (International Manufacturing Strategy Survey) sur un échantillon de 556 entreprises issues de secteurs industriels variés (Sun, 2000). Bien que Tan et Vonderembse (2006) notent que l'usage de ces TIC peut favoriser les liens dans les équipes projets et la coordination de leurs tâches, ce lien n'a pas encore été mis en évidence empiriquement. D'où notre hypothèse :

\section{Hypothèse $n^{\circ} 2:$ "L'utilisation des} TIC d'aide à la conception dans les équipes projets de NPD influence positivement l'efficacité de la coordination »

Au cours de ces dernières années, les TIC ont joué un rôle de plus en plus important comme support des communications des équipes projets de NPD (Nambisan, 2003). L'usage de ces TIC permet à leurs membres "de combiner, d'analyser, et de disséminer les informations relatives aux tâches, ${ }^{2}$ (Ozer, 2000, p. 388). Selon Durmusoglu et Barczak
(2011, p. 322), l'usage des TIC permettant de "faciliter, d'intensifier ou d'étendre les interactions et la communication entre les acteurs pendant l'exécution des tâches de NPD ${ }^{3}$. Plusieurs recherches ont montré empiriquement qu'en facilitant l'organisation et la synchronisation des informations au cours des processus de NPD, l'usage de ces TIC facilite la performance des projets de NPD (Barczak et al. 2008 ; Durmusoglu et Barczak, 2011).

Au cours des projets, les courriels et le téléphone sont les TIC les plus fréquemment utilisées pour communiquer (Pauleen et Yoong, 2001) ainsi que les outils de réunions en ligne (Durmusoglu et Barczak, 2011). L'usage des TIC de communications synchrones permettant l'usage de la voix et/ou de l'image est ainsi privilégié (Chidambaram et Jones, 1993 ; Sosa et al. 2002). Ces TIC permettent de transférer davantage d'informations que les TIC asynchrones car elles empruntent de nombreux canaux grâce à l'intonation de la voie et/ou aux expressions du visage (Chidambaram et Jones, 1993 ; Patrashkova et McComb, 2004). Toutefois, la démarche quantitative de Patrashkova et McComb (2004) a démontré que, audelà d'une certaine fréquence d'usage des TIC de communications synchrones dans les équipes de NPD, il y a une surcharge informationnelle. Leur étude quantitative lie négativement cette surcharge à la satisfaction de l'équipe. Toutefois, nous proposons d'examiner la

\footnotetext{
2 "In general, IT includes computer hardware, software, and communication systems, (...) used to facilitate the compilation, analysis, and dissemination of task-related information" (Ozer, 2000, p. 388).

3 "Computer-mediated communication technologies are IT tools that facilitate, intensify or expand the interaction of and communication between employees during NPD task executions" (Durmusoglu et Barczak, 2011, p. 322).
} 
relation entre l'usage des TIC pour communiquer de façon synchrone dans les équipes projets de NPD et la coordination des tâches en posant l'hypothèse suivante :

\section{Hypotbèse $n^{\circ} 3$ : "L'utilisation} fréquente des TIC de communications synchrones dans les équipes projets de NPD influence positivement l'efficacité de la coordination"

Si l'usage des TIC dans les équipes de NPD devient de plus en plus fréquent (Nambisan, 2003), les échanges en présentiel ne semblent pas pour autant abandonnés, car ils constitueraient un élément essentiel dans le processus de socialisation des équipes. L'étude ethnographique de Nardi et Whittaker (2002) auprès de 22 personnes a permis d'identifier les bénéfices du présentiel dans ce processus de socialisation. Au cours de ces échanges en présentiel, les interlocuteurs se sentent connectés en partageant un espace commun, une simple poignée de mains ou un regard pouvant renforcer cette connexion. Aussi certains auteurs avancent-ils que les communications médiatisées par les TIC atteignent difficilement le même niveau de richesse médiatique que les échanges en présentiel (Allen et Henn, 2006). Par " richesse des médias ", Daft et Lengel (1986) décrivent les capacités des médias pour transférer des signes, du feedback et des informations. Cette approche théorique part du principe que les interactions réalisées en présentiel constituent la forme médiatique la plus riche pour coordonner un travail car elles véhiculent une multitude de signaux visuels et langagiers. À l'inverse, les interactions au travers de l'échange de documents écrits véhiculeraient peu de signaux. Dans le cadre d'une étude de cas sur des équipes de NPD, Salter et Gann (2003) montrent que les échanges en présentiel sont essentiels pour résoudre des problèmes, trouver de nouvelles idées et évaluer la qualité du travail accompli, l'usage des TIC étant un complément du présentiel - et non un substitut.

De la même manière, la littérature sur la coordination des tâches semble également assez controversée à ce sujet. Si les travaux initiaux (Thompson, 1967) indiquent que les ajustements mutuels reposent sur des échanges en présentiel, une étude de cinq services bancaires distribués montre au contraire que les ajustements mutuels peuvent être réalisés grâce aux TIC (Michaux, 2005). La téléprésence pourrait donc se substituer aux échanges en présentiel (Schultze et Orlikowski, 2001). Ces études tendent ainsi à mettre en défaut la théorie de la richesse des médias pour expliquer l'efficacité de la coordination.

Dans cette lignée, Carlson et Zmud (1999) enrichissent le modèle initial de Daft et Lengel (1986) en montrant que les effets positifs attendus de l'usage de médias riches ne reposent pas uniquement sur les dimensions intrinsèques des supports mais également sur l'expérience individuelle et collective de ces médias. Dans la même veine, l'approche herméneutique met l'accent sur cette expérience, notamment sur le sens que l'usager confère à ces médias (Lee, 1994). L'expérience et le sens donné aux supports pourraient expliquer que l'usage de certains médias, dits moins "riches " au sens de Daft et Lengel (1986), suffisent à une bonne coordination. Nous proposons d'examiner empiriquement la relation entre 
présentiel et coordination dans les équipes de NPD avec l'hypothèse suivante :

\section{Hypotbèse $n^{\circ} 4$ : "Des communica-} tions fréquentes en présentiel dans les équipes projets de NPD influencent positivement l'efficacité de la coordination »

Après avoir examiné les différents types de médias pour coordonner des tâches, nous étudions l'efficacité de ces médias lorsque les équipes projets sont distribuées.

\section{L'EFFET MODERATEUR DU NIVEAU DE DISTRIBUTION DES ÉQUIPES}

La distribution des équipes prend la forme d'un continuum (Bierly et al. 2009 ; Kim et Kim, 2009) allant des équipes localisées aux équipes très distribuées dont les membres sont répartis autour du globe sur plusieurs fuseaux horaires et qui peuvent avoir plus de mal à se rencontrer (Griffith et Neale, 2001). Plusieurs recherches ont comparé les équipes projets localisées avec les équipes projets distribuées (Hoegl et al. 2007 ; Martins et al. 2004). L'étude de 145 projets de NPD d'Hoegl et al. (2007) démontre que, plus l'équipe est distribuée géographiquement, plus le besoin de coordination est important faute de quoi la performance des projets de NPD se dégrade. Toutefois, la coordination des tâches des équipes distribuées de NPD est considérée comme plus délicate que celle des équipes localisées du fait même de la distance (Espinosa et al. 2003). En ces termes, plus le niveau de distribution est élevé, plus la coordination des tâches semblerait difficile (Bierly et al. 2009 ; Cummings et al. 2009 ; McDonough et al. 2001). Les équipes projets de NPD distribuées cumulent ainsi un plus grand nombre de difficultés de coordination que les équipes projets localisées, difficultés qui exercent des effets négatifs sur la performance desdits projets (Cummings et al. 2009 ; McDonough et al. 2001).

Comme la distribution des équipes diminue les occasions d'échanger en présentiel (Fiol et O'Connor, 2005 ; Kirkman et Mathieu, 2005), elles utilisent d'autres médias pour se coordonner. Aussi la distribution des équipes projets de NPD entraîne-t-elle une dépendance à l'usage des TIC pour communiquer et traiter les informations (Cramton, 2001 ; Hertel et al. 2005). Plus les membres des équipes de NPD sont distribués, plus ils dépendraient de l'usage des TIC pour échanger des informations (Boutellier et al. 1998 ; Barczak et al. 2008).

Pour Schmidt et al. (2001), l'efficacité des décisions prises par des groupes d'étudiants distribués et médiatisés par les TIC serait meilleure que celle de groupes localisés. Par ailleurs, les TIC offriraient une flexibilité et une fiabilité suffisantes pour traiter les informations nécessaires à la coordination des tâches pour les équipes projets de NPD distribuées (Hameri et Nihtilä, 1997).

Dans le cadre des équipes distribuées, l'usage des TIC pour organiser les tâches peut faciliter la diffusion et la fiabilité des informations partagées (Boutellier et al. 1998). Il s'avère utile dans la mesure où il est nécessaire de planifier et de visualiser le travail de chacun, une planification plus détaillée permettant à chacun de repérer les ef- 
forts de mise en relation entre les sites et de déceler les besoins de coordination liés à l'interdépendance des taches (Cummings et al. 2009). La décomposition des tâches diminue aussi le risque d'erreurs en anticipant le travail de chacun des sites du projet (Kiesler et Cummings, 2002). Baba et Nobeoka (1998) ont montré les bénéfices de l'usage de ces technologies en étudiant les logiques de coordination au cours du développement du Boeing 777. Dans ce projet, plus de 250 équipes ont fait un usage intensif de cette technologie, qui a permis de minimiser les changements et les retours en arrière de 75\% par rapport aux projets précédents.

Par ailleurs, l'usage des TIC d'aide à la conception peut renforcer les liens entre les membres de l'équipe distribuée (Subrahmanian et al. 2003). Des technologies de type CAO réduisent le besoin d'interactions interindividuelles au sein de l'équipe tout en favorisant la coordination des tâches (Eisenhardt et Tabrizi, 1995 ; Gerwin et Barrowman, 2002). Cette réduction du besoin d'interaction serait utile aux équipes distribuées qui ne peuvent avoir une forte fréquence d'échanges en présentiel.

En outre, l'usage des TIC de communications synchrones permettrait de diminuer les effets négatifs de la distance sur le partage d'informations dans l'équipe et faciliterait ainsi la coordination des tâches (Chidambaram et Jones, 1993). Sosa et al. (2002) notent que, au cours des projets de NPD, les échanges en présentiel peuvent être remplacés par l'usage des TIC de communications synchrones lorsque la distance augmente. Toutefois, ces éléments n'ont pas été testés empiriquement à notre connaissance. En somme, au fur et à mesure que le niveau de distribution des équipes augmente, la fréquence d'usage des TIC s'intensifierait (Griffith et Neale, 2001). Néanmoins, l'ensemble des travaux traitant de la coordination par les TIC n'ont pas pris en considération l'efficacité de ces médias selon le niveau de distribution des équipes de NPD. Dans la lignée des travaux sur le rôle modérateur de la distribution géographique des équipes de NPD sur l'impact de leur coordination au regard de la performance des projets (Hoegl et al. 2007 ; Salomo et al. 2010), nous examinons le rôle modérateur du niveau de distribution des équipes NPD sur l'efficacité des médias de coordination. D'un point de vue théorique, nous nous appuyons sur les travaux de Lee (1994) notant que l'expérience des médias dans les équipes peut varier selon le contexte dans lequel ils sont utilisés. Le niveau de distribution des équipes pouvant être entendu comme un facteur contextuel (Boutellier et al. 1998 ; Hoegl et al. 2007 ; Salomo et al. 2010) :

H5 : "L'effet positif des TIC a. pour l'organisation des tâches, $b$. d'aide à la conception, c. de communications synchrones sur l'efficacité de la coordination est amplifié lorsque la distribution des équipes augmente »

Assez logiquement, certains travaux réalisés sur les équipes de NPD ont souligné les possibilités de réaliser l'intégralité du travail au travers des TIC uniquement. L'étude de cas de Malhotra et al. (2001) donne l'exemple d'une équipe de NPD ayant réalisé un projet dans les temps dans le domaine de l'aérospatial sans jamais se rencontrer en présentiel. D'autres travaux notent que malgré la distance qui sépare les 
membres des équipes projets le rôle des échanges en présentiel serait primordial pour coordonner efficacement les tâches (Orlikowski, 2002, Warkentin et al. 1997). Dans les équipes distribuées, les échanges en présentiel ont généralement lieu au début du projet au moment de la formation de l'équipe - puis de façon périodique au cours du projet (Kirkman et al. 2004). En comparant des groupes d'étudiants, Warkentin et al. (1997) montrent que la force des relations au sein des groupes agit positivement sur la qualité de partage des informations. Aussi des échanges occasionnels en présentiel peuvent-ils renforcer les relations au sein des équipes médiatisées par les TIC, et favoriser le partage d'informations et la coordination des tâches. Selon Fiol et O'Connor (2005), la diminution de fréquence du présentiel à distance peut influencer l'expérience de ce média dans les équipes distribuées. Contrairement à
Fiol et O'Connor (2005), qui assimilent projets localisés et présentiel, nous faisons l'hypothèse que les échanges en présentiel ont leur pertinence dans le travail des équipes projets, y compris lorsqu'ils sont fortement distribués :

H6 : "L'effet positif des communications en présentiel sur l'efficacité de la coordination est amplifié lorsque la distribution des équipes augmente »

Le modèle conceptuel est schématisé de la façon suivante :

\section{MÉTHODOLOGIE}

\section{III.1. Terrain et données}

Les recherches empiriques existantes étudient surtout les comportements de groupes d'étudiants pour examiner les

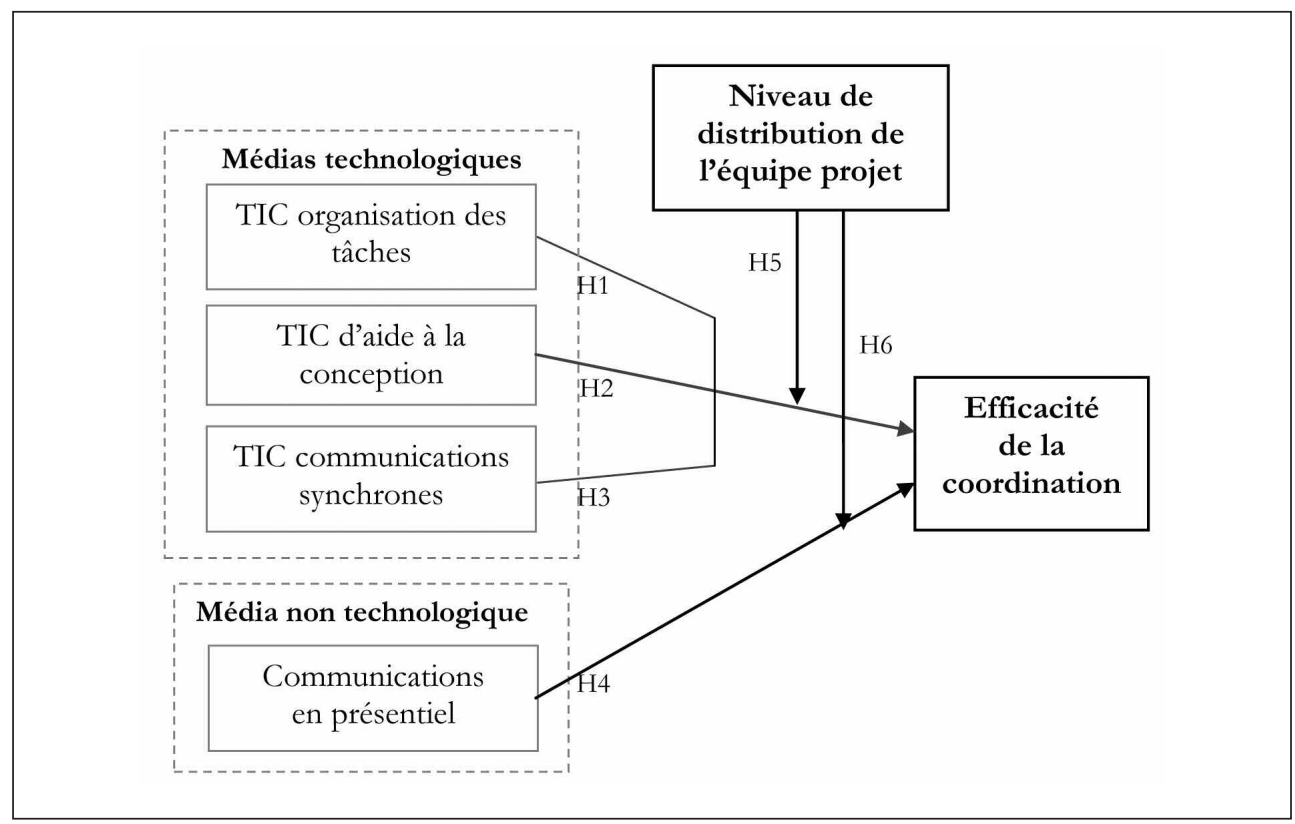

Schéma 1 : Le modèle de recherche 
effets de la distribution spatio-temporelle (Bierly et al. 2009 ; Martins et al. 2004). La transposabilité des résultats est toutefois limitée (Bierly et al. 2009), ce qui réduit la validité externe de ces recherches. Par conséquent, nous choisissons d'étudier la coordination des tâches d'équipes projets de NPD en situation " réelle " dans les secteurs manufacturiers, curieusement peu étudiée empiriquement (Kotlarsky et al. 2008 ; McDonough et al. 2001). Par ailleurs, nous choisissons d'interroger tous les membres des équipes projets (Akgün et al. 2010 ; Hoegl et Gemuenden, 2001) afin de pallier le manque de visibilité probable des responsables des projets sur les comportements de l'équipe distribuée dans son ensemble (Langevin, 2002 ; Ozer, 2000). Nous recueillons donc la perception de l'ensemble des membres quelles que soient leurs positions géographique et hiérarchique.

Nous avons toutefois pris un certain nombre de précautions dans la sélection des membres de ces équipes projets. Dans la lignée de Clift et Vandenbosch (1999) et Gupta et al. (2009), nous faisons le choix de collecter les réponses d'individus ayant participé à des projets de NPD similaires et facilement comparables. Nous focalisons notre recherche sur les membres d'équipes ayant participé à des projets de NPD relativement complexes, ceux-ci comportant davantage de difficultés de coordination (Mihm et al. 2003). En effet, de taille souvent importante, ils présentent en général des degrés d'innovation et d'interdépendance des tâches élevés
(Dvir et al. 1998 ; Mihm et al. 2003). Ces similarités permettront d'examiner des équipes projets ayant été confrontées à des tensions de coordination et permettront de distinguer l'impact du niveau de distribution des équipes sur la coordination des tâches.

\section{III.2. Sélection des équipes projets}

Les membres des équipes projets susceptibles de correspondre aux caractéristiques ciblées ont été sélectionnés en utilisant le réseau professionnel en ligne Viadeo. Nous avons contacté des directeurs ou chefs de projet, des responsables de bureau d'étude et des ingénieurs dans les secteurs de l'aéronautique, de l'automobile et des équipements industriels.

Nous avons obtenu l'accord de 15 responsables de projets de NPD déjà terminés pour participer à la recherche. Tout comme Srivastava et al. (2006), nous avons demandé à ces responsables de projet de diffuser l'enquête auprès des membres de leur ancienne équipe. Par ailleurs, afin de calculer les indices de distribution des membres des équipes, ils ont été sollicités pour donner les noms des villes de localisation des sites du projet ainsi que le nombre d'acteurs ayant participé au projet par site. La collecte des données a été réalisée grâce à la diffusion du questionnaire en ligne ${ }^{4}$. Avant de diffuser l'enquête, un pré-test a été réalisé. Dix acteurs projets ont participé à l'évaluation de la qualité du questionnaire.

${ }^{4}$ Nous avons utilisé le logiciel Sphinx online pour cette étape. 
Les remarques obtenues ont permis de reformuler la question 23. À l'issue de cette étape, nous avons validé notre questionnaire.

Les répondants ont été sollicités par e-mail par les responsables des projets. Compte tenu de la dimension multiculturelle des équipes distribuées, deux liens hypertextes ont été proposés à chaque répondant (versions française et anglaise, qui a été traduite par un professionnel). La diffusion du questionnaire s'est déroulée sur 3 mois entre octobre et décembre 2011. Parmi les 15 responsables, 7 ont obtenu des réponses de plusieurs membres de leur équipe. Ces 7 projets retenus sont relativement similaires au regard de leur taille, du niveau d'interdépendance des taches, du niveau d'innovation du produit développé et du secteur (cf. Annexe A). L'ensemble des membres de ces 7 équipes projets représente un effectif de 209 personnes. Parmi ces 209 personnes, 153 ont été sollicitées par les responsables projets. Les autres membres de ces projets n'ont pu être contactés car ils avaient changé de fonction ou d'entreprise. En définitive, 88 questionnaires ont été retournés, ce qui correspond à un taux de réponse très satisfaisant de 57,5\%.

\section{III.3. Les variables}

Pour tester les hypothèses, nous retenons différentes mesures issues de la littérature. Chaque variable est mesurée grâce à une échelle de Likert en 5 points allant de "pas du tout d'accord" à " tout à fait d'accord ". Le questionnaire figure en Annexe B.

\section{- Variable dépendante : l'efficacité de la coordination des équipes}

La majorité des mesures se focalise sur la coordination des tâches des membres des équipes marketing et ingénierie (Pinto et Pinto, 1990). Nous proposons d'élargir notre mesure à tous les métiers de l'équipe. Cette approche globale permet de mieux rendre compte du travail des équipes interfonctionnelles (Pinto et al. 1993). Nous évaluons l'efficacité de la coordination à partir de l'échelle de mesure précédemment validée par Leenders et Wierenga (2002), qui évalue la coordination à partir de la qualité des communications, de la collaboration et de la coopération dans les équipes. Nous complétons cette échelle avec les mesures du glitch proposées par Hoegl et Gemuenden (2001). En effet, certains auteurs (Olson et al. 2001 ; Hoopes et Postrel, 1999) notent qu'une coordination efficace est plus difficile à évaluer que les problèmes de coordination. Avec le concept du glitch, que l'on peut résumer par " l'absence de partage d'informations et de connaissances ", Hoopes et Postrel (1999) mettent en évidence que les projets dont le niveau de glitch est élevé se coordonnent mal. En définitive, sept indicateurs issus de la littérature ont été retenus (cf. Annexe B).

\section{— Variables indépendantes}

Notre objectif est de mesurer la fréquence d'usage de certains médias technologiques pour coordonner les tâches dans les équipes projets de NPD. Une première démarche exploratoire de nature qualitative à partir de 19 entretiens réalisés avec des responsables R\&D et chefs de projets de NPD distri- 
bués nous a permis d'identifier les TIC les plus fréquemment utilisées dans ces équipes (en leur suggérant CAD/CAM, PLM, MS-Project, la visioconférence, les conférences téléphoniques, etc.). Cette démarche nous a conduits à écarter la technologie PLM, très rarement utilisée par les interlocuteurs de notre échantillon. Nous distinguons ainsi trois types de TIC en fonction de leurs fonctionnalités dans les projets de NPD : TIC synchrones, TIC d'aide à la conception, TIC pour organiser les tâches que nous détaillons ci-dessous.

Fréquence d'usage des TIC synchrones: nous utilisons d'une part l'indicateur employé par Chidambaram et Jones (1993) dans le cadre d'une étude portant sur la qualité des décisions prises par les équipes distribuées, qui a trait à l'usage des conférences téléphoniques. D'autre part, d'autres TIC de communications synchrones étant utilisées au sein des équipes distribuées (cf. liste réalisée par Boutellier et al. 1998), nous ajoutons deux autres indicateurs afin d'étudier l'ensemble des TIC de communications synchrones - et non une technologie particulière, liés à l'usage du téléphone et de la visioconférence (Boutellier et al. 1998). Les trois indicateurs reflètent les variations de l'utilisation des TIC de communications synchrones et sont de type réflexif.

Fréquence d'usage des TIC d'aide à la conception : un indicateur unique est utilisé à partir de Pavlou et El Sawy (2006).

Fréquence d'usage des TIC pour organiser les tâches : trois indicateurs permettent d'offrir une description claire de ces technologies aux répondants. Nous souhaitons les accompagner en citant certains exemples comme le logiciel "MS-Project". Les indicateurs proposés reflètent la variable et constituent donc des indicateurs réflexifs.

Fréquence des communications réalisées en présentiel: un indicateur unique est utilisé, dans la lignée des travaux dans le cadre des équipes distribuées de Hertel et al. (2004).

\section{- Variable modératrice : la dis- tribution spatio-temporelle}

Nous faisons le choix d'étudier les distances " objectives " spatiale et temporelle car ce sont les plus fréquemment citées dans les recherches sur la coordination des équipes projets distribuées (Alfaro, 2014 ; Cummings et al., 2009 ; Espinosa et al., 2003 ; Hertel et al., 2004 ; O'Leary et Cummings, 2007). Dans la lignée des travaux d'Espinosa et al. (2003) nous limitons ainsi le nombre de distances étudiées afin d'éviter la confusion de leurs effets respectifs. Les distances dites "subjectives " ont un impact important mais peuvent évoluer tout au long du projet (Watson-Manheim et al., 2011), ce qui peut nuire à la stabilité de la mesure.

En conséquence, nous retenons la mesure objective du niveau de distribution spatio-temporelle proposée par O'Leary et Cummings (2007). Ces auteurs mesurent la distance kilométrique moyenne et le nombre moyen de fuseaux horaires qui séparent les membres des équipes projets. Le calcul des différents indices pondère la distance moyenne en fonction du nombre de membres que comporte chaque équipe. En outre, le nombre de sites est pris en considération, car leur multiplication 
augmente le niveau de distribution (O'Leary et Cummings, 2007). Les formules de calcul de l'indice de distribution sont données en annexe $\mathrm{B}$ et leurs résultats en annexe $\mathrm{C}$. Les trois indicateurs obtenus sont considérés comme interchangeables et intercorrélés. En effet, l'augmentation de la distance géographique peut s'accompagner d'une variation de la distance temporelle. Ainsi, ces variables donnent lieu à un construit de type réflexif.

\section{— Variables de contrôle}

Nous ajoutons deux variables de contrôle au modèle de recherche. Selon le niveau d'interdépendance des tâches, le besoin de coordination varie de manière importante (Sosa et al. 2004). Même si nous avons pris soin d'étudier des projets de secteurs et de natures proches, il est important de vérifier que ces derniers ne reposent pas sur des tâches modulaires, réduisant ainsi le besoin de coordination (Sanchez et Mahoney, 1996). La mesure de l'interdépendance des tâches est une variable latente réflexive adaptée de Pearce et Gregersen (1991). Par ailleurs, dans la mesure où notre étude porte sur les membres des équipes projets, il est important de contrôler que l'appartenance à une équipe en particulier n'influence pas la coordination. Cette variable ne joue ici qu'un rôle confirmatoire car l'homogénéité des projets a été contrôlée en amont par les critères ayant servis à la sélection des projets (cf. Annexe A).

\section{III.4. Echantillon}

Les membres des équipes projets interrogés travaillent dans les industries de l'aérospatiale, de la microélectronique, des télécommunications, des équipements industriels et de l'automobile, et de la mécanique. Ces membres font partie d'équipes projets de taille moyenne : plus de 20 personnes avec une moyenne de 30 personnes et un écart-type de 8 personnes sur les 7 projets (cf. Annexe A). En moyenne, 2296 kilomètres et 1,5 fuseau horaire séparent les membres de ces équipes (cf. Annexe C), qui sont réparties sur 4 sites différents en moyenne. Les écarts-types des indices de distribution spatiale et temporelle sont élevés et proches de leur moyenne, ce qui va dans le sens de la variabilité recherchée sur ce critère. Afin d'obtenir une variable avec une métrique similaire à celle des autres variables du modèle, nous transformons les indices obtenus en une variable numérique discrètes. Pour obtenir une métrique sur 5 points, nous avons procédé à un regroupement visuel pour chacun des indices obtenus. Cette transformation permet d'obtenir des indices mesurés sur une échelle allant de 1 à 5 pour chacun des indicateurs de distribution. Le principe de cette transformation est de scinder en 5 parties l'intervalle de valeurs obtenues sur la variable continue des indices de distribution.

Afin d'écarter les possibilités de covariances biaisées dans les données (Rönkkö et Ylitalo, 2011 ; Podsakoff et Organ, 1986), nous avons réalisé un test

\footnotetext{
${ }^{5}$ Opération réalisée avec le logiciel SPSS.
} 
d'Harman ${ }^{6}$ avec l'ensemble des variables du modèle. La covariance expliquée par le facteur unique est de $23,86 \%$ pour la première composante, indiquant que les biais de méthode ne semblent pas significatifs.

\section{RÉSULTATS}

Pour analyser les résultats, nous utilisons les équations structurelles de type PLS (Partial Least Squared). Cette méthode convient particulièrement à nos objectifs de recherche car elle est de type prédictif et permet d'examiner des phénomènes encore peu étudiés (Esposito Vinzi et al. 2010 ; Hair et al. 2011 ; Sosik et al. 2009). Elle repose sur la prédiction des variables expliquées dont l'objectif est de maximiser la variance expliquée et de minimiser les variances résiduelles (Chin et Dibbern, 2010). Nous procédons dans un premier temps à la validation des mesures utilisées, puis poursuivons par le test des hypothèses.

\section{IV.1. Qualité des mesures des variables}

Afin de nous assurer de la validité des mesures, nous testons en premier lieu le modèle structurel externe. Dans cette démarche, nous avons supprimé les indicateurs réflexifs qui n'étaient pas suffisamment corrélés avec leur variable de référence. Le seuil de corrélation retenu est de 0,5 au regard des critères conformes à une recherche prédictive. En outre, nous testons la significativité de ces corrélations par ré-échantillonnage selon la méthode du bootstrap (avec 500 itérations). À l'issue de ce test de fiabilité des indicateurs, nous contrôlons la consistance interne avec l'indice de fiabilité composite (CR, Composite Reliability). Nous examinons l'alpha de Cronbach à titre informatif car, en dessous de 3 variables, la fiabilité de cet indicateur n'est pas avérée et l'indice de fiabilité composite prime dans le cadre des modèles PLS (Chin et al. 2003 ; Tenenhaus et al. 2005). La valeur de ces indices est jugée satisfaisante à partir de 0,6 (Esposito Vinzi et al. 2010). La validité convergente est évaluée en fonction de la variance moyenne extraite de la variable latente, qui doit atteindre au moins $50 \%$. Le tableau 2 présente les variables composites réflexives, variables dont on peut évaluer la consistance interne et la validité convergente, les variables mono item n'étant testées qu'au niveau de leur validité discriminante.

La matrice des corrélations montre que les liens entre les variables ne sont pas élevés et démontre de ce fait l'indépendance théorique des variables (Tableau 3). Au regard des résultats des tableaux 2 et 3 , les validités convergente et discriminante sont donc vérifiées pour l'ensemble des variables du modèle.

En outre, l'analyse des corrélations croisées entre les différents indicateurs atteste de la validité discriminante des variables avec un indicateur unique au seuil de 0,6 (cf. Annexe D). À l'issue de cette validation du modèle structurel

${ }^{6}$ Test réalisé avec le logiciel "SPSS ". 
Tableau 2 : Fiabilité des mesures et validité convergente

\begin{tabular}{|c|c|c|c|c|c|}
\hline & $\begin{array}{l}\text { Variables } \\
\text { latentes }\end{array}$ & $\begin{array}{l}\text { Variables } \\
\text { manifestes }\end{array}$ & Corrélations & Fiabilité & AVE \\
\hline $\begin{array}{l}\text { Variables } \\
\text { expliquées }\end{array}$ & $\begin{array}{l}\text { Efficacité } \\
\text { de la } \\
\text { coordination }\end{array}$ & $\begin{array}{c}\text { COORD_1 } \\
\text { COORD_2 } \\
\text { COORD_3 } \\
\text { COORD_4 } \\
\text { R_COORD_5 } \\
\text { R_COORD_6 } \\
\text { R_COORD_9 } \\
\end{array}$ & $\begin{array}{l}0,799^{* * *} \\
0,731^{* * * *} \\
0,820^{* * * *} \\
0,781^{* * *} \\
0,620^{* * *} \\
0,605^{* * * *} \\
0,643^{* * *} \\
\end{array}$ & $\begin{array}{c}\mathrm{CR}: 0,881 \\
\alpha: 0,844\end{array}$ & 0,517 \\
\hline Modérateur & $\begin{array}{l}\text { Distribution } \\
\text { de l'équipe }\end{array}$ & $\begin{array}{l}\text { DIST_SPA_O } \\
\text { DIST_TEMP_O }\end{array}$ & $\begin{array}{l}0,965^{* * * *} \\
0,971^{* * * *}\end{array}$ & CR : 0,967 & 0,937 \\
\hline $\begin{array}{l}\text { Variables } \\
\text { explicatives }\end{array}$ & $\begin{array}{l}\text { TIC organisation } \\
\text { des tâches }\end{array}$ & $\begin{array}{l}\text { TIC_5_ORGA } \\
\text { TIC_6_ORGA } \\
\text { TIC_7_ORGA }\end{array}$ & \begin{tabular}{|l}
$0,865^{* * * *}$ \\
$0,590^{* * *}$ \\
$0,851^{* * *}$
\end{tabular} & $\begin{array}{l}\text { CR : } 0,819 \\
\alpha: 0,7033\end{array}$ & 0,607 \\
\hline $\begin{array}{l}\text { Variables } \\
\text { de contrôle }\end{array}$ & Interdépendance & $\begin{array}{c}\text { R_INTER_TA_1 } \\
\text { INTER_TA_2 }\end{array}$ & $\frac{0,914^{* * *}}{0,668^{* *}}$ & $\mathrm{CR}: 0,777$ & 0,641 \\
\hline Test un & Bootstrap $(500$ & $* * *<<0,001 \quad(3$, & $\mathrm{p}<0,01(2,3$ & $0,0)(1,0$ & $(1,25)$ \\
\hline
\end{tabular}

Tableau 3 : Validité discriminante des variables du modèle

\begin{tabular}{|c|l|c|c|c|c|}
\hline & & 1 & 2 & 3 & 4 \\
\hline 1 & Efficacité de la coordination & $\mathbf{( 0 , 7 2 )}$ & 0 & 0 & 0 \\
\hline 2 & Interdépendance des tâches & 0,23 & $\mathbf{( 0 , 8 0 )}$ & 0 & 0 \\
\hline 3 & Distribution de l'équipe projet & $-0,26$ & $-0,08$ & $\mathbf{( 0 , 9 7 )}$ & 0 \\
\hline 4 & TIC organisation des tâches & 0,33 & 0,12 & $-0,08$ & $\mathbf{( 0 , 7 8 )}$ \\
\hline
\end{tabular}

externe, nous procédons à l'analyse du modèle interne.

\section{IV.2. Analyse du modèle interne}

Afin d'obtenir un résultat statistique stable, il est préférable de fixer le nombre de réplications de l'échantillonnage à 500 (Marcoulides et Saunders, 2006). Le test du modèle interne s'articule autour de deux modèles. Dans le premier, nous étudions uniquement les effets directs des médias utilisés sur l'efficacité de la coordination ; dans le second, nous examinons à la fois les effets directs et l'effet indirect du niveau de distribution sur la relation entre les médias et la coordination. Le modèle 2 permet de restituer $42 \%$ de variance expliquée et témoigne de l'importance du pouvoir explicatif de l'effet modérateur de la distribution, l'effet d'interaction améliore le modèle de près de 20\% de variance supplémentaire par rapport au modèle 1. Par ailleurs, les indices GoF et $\mathrm{Q}^{2}$ démontrent un bon niveau de fiabilité du modèle structurel (Tenenhaus et al. 2005). En tenant compte des seuils critiques pour des petits échantillons (Wetzels et al. 2009), l'indice GoF de notre modèle est de 0,38 bien supérieur au seuil critique de 0,1 et l'indice $Q^{2}$ est différent de zéro $\left(Q^{2}: 0,171\right)$. En conséquence, nous retenons le modèle 2 pour l'analyse. 
Tableau 4 : Résultats du modèle structurel interne

\begin{tabular}{|c|c|c|}
\hline & Modèle 1 & Modèle 2 \\
\hline & $\beta$ & $\beta$ \\
\hline \multicolumn{3}{|l|}{ Variables contrôle $\rightarrow$ Efficacité de la coordination } \\
\hline - Interdépendance des tâches & $0,175 \dagger$ & 0,102 \\
\hline - Projet & $-0,038$ & $-0,021$ \\
\hline \multicolumn{3}{|l|}{ Médias $\rightarrow$ Efficacité de la coordination } \\
\hline - TIC organisation des tâches (H1) & $0,244^{* *}$ & $0,144 \dagger$ \\
\hline - TIC aide à la conception (H2) & 0,065 & 0,117 \\
\hline - TIC communications synchrones (H3) & $-0,176 \dagger$ & $-0,129$ \\
\hline - Communications en présentiel (H4) & $0,239^{*}$ & $0,302^{* *}$ \\
\hline Distribution $\rightarrow$ Efficacité de la coordination & & $-0,148^{*}$ \\
\hline \multicolumn{3}{|l|}{ Effets d'interaction $\rightarrow$ Efficacité de la coordination } \\
\hline - TIC organisation des tâches * Distribution (H5a) & & $0,174^{*}$ \\
\hline - TIC aide à la conception * Distribution (H5b) & & $0,256^{* *}$ \\
\hline - TIC communications synchrones * Distribution ( $\mathrm{H} 5 \mathrm{c})$ & & 0,100 \\
\hline - Communications en présentiel * Distribution (H6) & & $-0,265^{*}$ \\
\hline $\mathbf{R}^{2}$ Efficacité de la coordination & 0,244 & 0,419 \\
\hline$\Delta \mathbf{R}^{2}$ & & 0,175 \\
\hline
\end{tabular}

\section{- L'influence directe des médias sur la coordination}

Dans l'échantillon, l'utilisation fréquente des TIC pour organiser les tâches influence positivement la coordination $(\beta=0,144, p<0,10)$. L'hypothèse $n^{\circ} 1$ est corroborée à 10\% d'erreur. L'utilisation des TIC de conception des produits ne contribue pas directement à l'efficacité de la coordination $(\beta=0,117$, ns.), infirmant ainsi l'hypothèse $\mathrm{n}^{\circ} 2$. Quant à l'utilisation des TIC de communications synchrones, elles n'influencent pas l'efficacité de la coordination des tâches $(\beta=-0,129$, ns.), infirmant également l'hypothèse $n^{\circ} 3$. En revanche, une fréquence importante des communications en présentiel parmi les membres des équipes projets de NPD étudiées agit très positivement sur l'efficacité de la coordination des tâches $(\beta=0,302, \quad p<0,01), \quad$ corroborant donc l'hypothèse $\mathrm{n}^{\circ} 4$.

\section{- Le rôle modérateur du niveau de distribution des membres des équipes}

Le niveau de distribution des équipes influence la relation entre les médias et la coordination des tâches. En effet, plus le niveau de distribution des membres des équipes projets de NPD augmente, plus l'effet positif de l'usage des TIC pour organiser les tâches s'intensifie $(\beta=0,174, p<0,05)$, ce qui va dans le sens de l'hypothèse $\mathrm{n}^{\circ} 5 \mathrm{a}$. Il en va de même de l'effet d'interaction entre la distribution et l'usage des TIC d'aide à la conception sur la coordination $(\beta=0,256, p<0,01)$ et de l'hypothèse $n^{\circ} 5$ b. En revanche, nous ne relevons pas d'effet d'interaction significatif entre 
le niveau de distribution et l'usage des TIC de communications synchrones sur l'efficacité de la coordination $(\beta=0,100$, ns.), résultat qui infirme l'hypothèse $\mathrm{n}^{\circ} \mathrm{c}$ c.

Les résultats pour la communication en présentiel montrent que plus le niveau de distribution est important, plus les réunions en présentiel jouent défavorablement sur la coordination $(\beta=-$ 0,265, $\mathrm{p}<0,05)$. En d'autres termes, la distribution a un impact d'autant plus négatif sur la coordination lorsque les équipes communiquent fréquemment en présentiel, ce qui infirme l'hypothèse $n^{\circ} 6$.

L'interdépendance des tâches n'influence pas l'efficacité de la coordination des tâches $(\beta=0,102$, ns.) ce qui peut signifier qu'il existe peu de différences de niveau d'interdépendance des taches entre les projets. En outre, l'appartenance à un projet spécifique n'exerce aucune influence significative sur la variable dépendante $(\beta=-0,030$, ns.) ce qui corrobore notre hypothèse de comparabilité des projets.

\section{DISCUSSION}

Cette recherche vise à identifier les médias favorables à la coordination des tâches des équipes projets de NPD distribuées. Nos résultats montrent que l'effet des médias sur la coordination des équipes dépend du niveau de distribution spatio-temporel des membres de l'équipe.

Premièrement, nos résultats montrent que la coordination des équipes peu distribuées repose essentiellement sur des échanges en présentiel et sur l'usa- ge des TIC pour organiser les tâches. Les résultats ne vont donc pas dans le sens d'un impact positif des TIC d'aide à la conception ni des TIC de communications synchrones sur la coordination des équipes de NPD peu distribuées. Les échanges en présentiel apparaissent primordiaux, alors que l'usage des TIC semble en retrait (2 TIC sur 3).

Deuxièmement, les résultats démontrent qu'au fur et à mesure que la distribution des équipes augmente, l'efficacité de leur coordination dépend de l'usage de médias technologiques. Plus précisément, les TIC d'aide à la conception et celles pour organiser les tâches favorisent la coordination des équipes très distribuées. Ceci apporte donc une corroboration empirique des travaux ayant souligné une dépendance à l'usage des TIC lorsque le niveau de distribution des équipes augmente (Barczak et al. 2008 ; Hertel et al. 2005).

Si l'on se tourne vers l'effet de chacun des médias étudiés, les TIC pour organiser les tâches facilitent la coordination des tâches dans les équipes projets de NPD - et ce quel que soit le niveau de distribution. Les résultats enrichissent les recherches antérieures en montrant que, lorsque le niveau de distribution augmente, l'utilisation de ces TIC doit également être plus importante (cf. test de pente du schéma 2). Ces résultats complètent l'étude de cas sur les projets très distants de Boutellier et al. (1998) dans laquelle ils suggèrent une relation positive entre l'usage des TIC pour organiser les tâches et la coordination des équipes distribuées. Nos résultats apportent une validation empirique à cette relation tout en prenant en consi- 
dération le niveau de distribution des équipes.

En ce qui concerne l'utilisation des TIC supports de la conception de produits, elle semble jouer un rôle clé sur la coordination dans les équipes projets très distribuées. Dans ce contexte, il est probable que les TIC de conception compensent les effets négatifs de la distribution sur la coordination $(\beta=-0,148$, $\mathrm{p}<0,05)$. Effectivement, l'effet d'interaction entre la distribution et les TIC d'aide à la conception donnant un résultat de signe positif, cette combinaison permettrait de contrecarrer l'effet négatif de la seule distribution sur la coordination. Certains auteurs ont montré que ces TIC d'aide à la conception peuvent limiter les besoins d'interactions (Gerwin et Barrowman, 2002) et les retours en arrière (Baba et Nobeoka, 1998). En facilitant les échanges d'informations sur le produit en cours de développement, ils permettent à l'équipe de franchir les frontières spatio-temporelles qui séparent les différents métiers (Durmusoglu et Barczak, 2011 ; Levina et Vaast, 2006) tout en renforçant les liens entre les différents métiers des équipes (Subrahmanian et al. 2003). Contrairement aux médias numériques, lorsque le niveau de distribution augmente, le recours au présentiel ne semble pas nécessaire. Nous avons vu que, lorsque que ce niveau de distribution est faible, les communications en présentiel améliorent la coordination projets de NPD (H4). Ces échanges sont l'occasion de prendre en considération le travail de tous les métiers de l'équipe et de partager des connaissances (Persaud, 2005 ; Orlikowski, 2002). En revanche, lorsque le niveau de distribution augmente, l'efficacité de la coordination diminue au fur et à mesure de l'augmentation des rencontres en présentiel.

L'explication de cette relation négative entre échanges en présentiel et coordination des équipes très distribuées (cf. Schéma 3) est délicate, car la majorité des recherches se concentre sur les avantages que procurent les communications en présentiel au sein des équipes (Oshri et al. 2007), les aspects

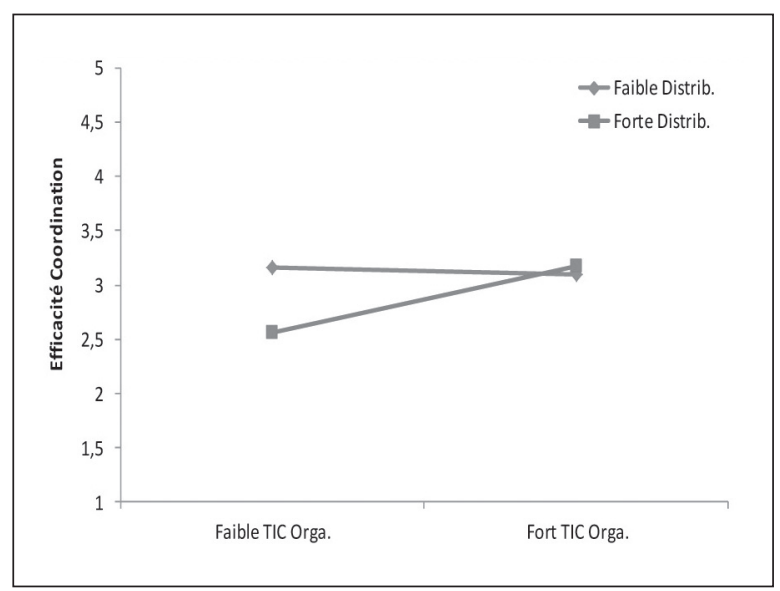

Schéma 2. Effet des TIC pour organiser les tâches sur la coordination selon le niveau de distribution 


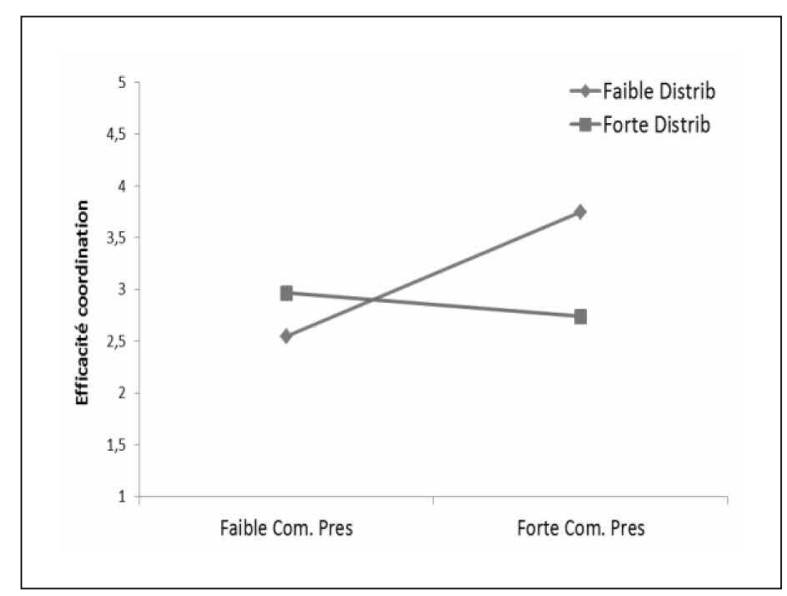

\section{Schéma 3 : Effet des communications en présentiel sur la coordination selon le niveau de distribution}

négatifs ayant fait l'objet d'un nombre de recherches beaucoup plus restreint.

Toutefois, l'étude de cas d'Oshri et al. (2007) montre que, dans un contexte de travail distribué, les échanges en présentiel sont souvent de courte durée. Ces courts moments d'échanges s'expliquent essentiellement par les coûts de coordination occasionnés par l'organisation de ces rencontres. En outre, la réduction de ces coûts passe par la sélection et la restriction du nombre de participants à ces échanges en présentiel. Ces choix pouvant s'avérer non pertinents, les avantages convoités initialement par ces communications s'en trouvent considérablement diminués. Par ailleurs, les auteurs remarquent que les échanges en présentiel dans les équipes distribuées portent essentiellement sur la résolution de problèmes techniques. Ces échanges n'ont donc pas nécessairement vocation à construire et à renforcer les liens au sein de l'équipe. Fiol et O'Connor (2005) soulignent à ce titre que la construction de ces liens interperson- nels permettant de construire une identité partagée s'avère problématique lorsque le niveau de distribution augmente. Or, nos résultats montrent que les échanges en présentiel ne permettent pas de soutenir ces liens dans les équipes très distribuées ni le transfert des informations entre les interlocuteurs. En somme, les bénéfices attendus des communications en présentiel requièrent du temps qui n'entraîne pas toujours des effets positifs sur l'efficacité de la coordination des projets très distribués (Nardi et Whittaker, 2002).

En montrant que des équipes fortement distribuées peuvent se coordonner en présentiel, nous contribuons également au débat sur la mesure du niveau de distribution des équipes. Certains auteurs caractérisent le niveau de distribution (ou de virtualité) des équipes selon la fréquence d'usage de certains supports de communication. Un faible niveau de distribution est appréhendé par des échanges fréquents en présentiel (Fiol et O'Connor, 2005 ; Griffith et Neale, 2001) et un niveau 
élevé est lié à une utilisation systématique des TIC (Griffith et al. 2003). Dans la lignée d'O'Leary et Cummings (2007), nous avons appréhendé le niveau de distribution par la distance spatio-temporelle des membres de l'équipe. Nos résultats indiquant que le niveau de distribution et les médias utilisés ne varient pas toujours dans le même sens, il apparait donc important de ne pas les confondre pour éviter des erreurs d'interprétation. En ce sens, notre recherche contribue également à clarifier le débat sur la définition du niveau de distribution des équipes.

\section{CONCLUSION}

Notre recherche a montré que, lorsque la distribution spatio-temporelle des équipes augmente, l'efficacité des médias technologiques pour se coordonner s'amplifie, notamment ceux permettant de coordonner les tâches (TIC d'organisation) et de résoudre des problèmes techniques collectifs (TIC de conception). Parallèlement, la coordination par l'usage du présentiel tend à diminuer l'efficacité de la coordination. Ce double effet rejoint des résultats antérieurs dans la littérature (Boutellier et al. 1998 ; Malhotra et al. 2001) : le fonctionnement des projets distants pourraient se passer de présentiel et reposer principalement sur les TIC. Nous apportons ici une corroboration empirique à cette littérature en testant l'effet de ces médias sur l'efficacité de la coordination d'équipes distribuées dans les projets de NPD.

La coordination de ces équipes semble reposer essentiellement sur des échanges relatifs aux tâches. En effet, l'efficacité de la coordination dépend essentiellement des médias dédiés à la conception du produit. Ce résultat va dans le sens des travaux de Kanawattanachai et Yoo (2007) qui montrent que les membres des équipes distribuées communiquent davantage sur les tâches sans nécessairement développer des relations interpersonnelles fortes.

Nos résultats ouvrent ainsi de nouvelles perspectives de recherche au sein du débat théorique portant sur la relation entre coordination à distance et présentiel. Contrairement à la littérature, nous montrons que cette relation n'est pas simple ni univoque. Nos résultats suggèrent que d'autres facteurs expliquent l'efficacité de la coordination à distance. La coordination des projets distribués n'est pas une version dégradée de la coordination des projets en présentiel. Au contraire, il semble reposer sur des mécanismes que nous avons partiellement identifiés et qui ouvre la voie à de nombreuses recherches futures.

L'examen de l'effet des TIC par catégorie (organisation, conception et synchrones) sur la coordination permet d'enrichir les résultats de Pavlou et El Sawy (2010), qui appréhendent les TIC dans leur globalité - et non de manière séparée. Aussi montrons-nous d'ailleurs que, selon leur nature, les TIC n'ont pas le même effet sur la coordination des équipes de NPD.

Cette recherche n'est pas exempte de certaines limites. La taille de l'échantillon ne permet pas de généraliser les résultats obtenus. Le recueil de données supplémentaires nous permettrait d'adopter une démarche confirmatoire en testant le modèle de recherche selon 
une modélisation structurelle de type maximum de vraisemblance. En outre, sachant que le présentiel n'a pas d'influence lorsque le niveau de distribution est élevé, l'absence d'effet des TIC de communications synchrones (visioconférence) sur la coordination quel que soit le niveau de distribution remet en question la théorie de la richesse des medias. Dans la lignée de Lee (1994) et Carlson et Zmud, (1999), nos résultats montrent que l'efficacité des médias pour coordonner les équipes distribuées n'est pas nécessairement liée à la richesse des médias. La notion de richesse relève sans doute davantage de l'usage, du sens et de l'expérience partagée de ces supports. En mobilisant une mesure de l'expérience partagée dans des travaux ultérieurs, il serait possible d'appréhender l'influence de la richesse des médias utilisés sur la coordination en mode distribué de manière à identifier quel type de TIC supporte ce processus (Marciniak et Rowe, 1999). Par ailleurs, la relation négative entre les communications en présentiel et l'efficacité de la coordination des équipes de NPD très distribuées pourrait être expliquée par une démarche qualitative en profondeur, des recherches complémentaires restant à mener sur le rôle du présentiel dans la coordination des projets de NPD, notamment distribués. Enfin, nous avons choisi une mesure objective de la distance spatiale et temporelle. Cette mesure aurait pu être complétée par le nombre de sites distants. Cette dimension est également objective et peut influencer le niveau de distribution lorsque le nombre de sites différents est élevé. En outre, même si elles sont moins stables et plus difficiles à appréhender, d'autres distances plus subjec- tives ont été relevées dans la littérature, cognitive (Pauleen et Yoong, 2001), épistémique (Espinosa et al. (2003) ou culturelle (Gibson et Gibbs, 2006). Elles pourraient sans doute modérer davantage l'effet de certains supports de communication sur la coordination. Par ailleurs, suivant Taylor et Spicer (2007), des approches complémentaires à celle de la distance en tant que mesure de l'espace organisationnel pourraient faire l'objet de recherches futures de nature qualitative longitudinale. En particulier, il pourrait être intéressant de prendre en compte l'espace en tant que matérialisation de relations de pouvoir au sein des équipes de NPD. Ces relations de pouvoir ont en effet une influence clé sur le fonctionnement des projets de NPD (Ancona et Caldwell, 1992).

D'un point de vue managérial, cette recherche s'adresse aux responsables de projets de NPD distribués soucieux d'améliorer la coordination de leurs équipes. Nos résultats incitent ces chefs de projets de NPD à prôner l'usage de certains médias (TIC pour organiser les tâches, TIC d'aide à la conception) pour compenser les effets négatifs d'une distribution importante des équipes sur la coordination et améliorer la performance des projets. Plus important encore, il semble que d'inciter les membres des équipes à se rencontrer physiquement dès lors qu'ils sont distants ne semble pas améliorer leur coordination, sauf comme le suggère la littérature en début de projet pour établir la relation (Bouillot, 1999). Ces décisions pouvant s'avérer coûteuses, on ne peut que suggérer aux responsables de projet distants d'être prudents avec ce type de média. 


\section{BIBLIOGRAPHIE}

Akgün A., Byrne J.C., Lynn G.S. et Keskin H. (2007), "Team stressors, management support, and project and process outcomes in new product development projects ", Technovation, Vol. 27, n¹0, p. 628-639.

Alfaro I. (2014) " Helping Global SoftwareDevelopment Teams to Overcome the Challenges of Temporal Dispersion and National Diversity: The Importance of Leadership Roles "Rowe F., Te'eni D. (Eds), Innovation and IT in an international context: RED strategy and operations, Palgrave MacMillan.

Allen T.J. et Henn G. (2006), "The organization and architecture of innovation: Managing the flow of technology ", Butterworth-Heinemann.

Ancona, D.G., Caldwell, D.F., (1992). Bridging the Boundary: External Activity and Performance in Organizational Teams. Administrative Science Quarterly, 37, 634665.

Baba Y. et Nobeoka K. (1998), "Towards knowledge-based product development: the 3-D CAD model of knowledge creation ", Research Policy, Vol. 26, n6, p. 643-659.

Banker R. D., Bardhan I. et Asdemir O. (2006), " Understanding the Impact of Collaboration Software on Product Design and Development. ", Information Systems Research, Vol. 17, n4, p. 352-373.

Barczak G., Hultink E. J. et Sultan F. (2008), " Antecedents and Consequences of Information Technology Usage in NPD: A Comparison of Dutch and U.S. Companies ", Journal of Product Innovation Management, Vol. 25, n6, p. 620-631.

Bierly P.E., Stark E.M. et Kessler E.H. (2009), "The Moderating Effects of Virtuality on the Antecedents and Outcome of NPD
Team Trust ", Journal of Product Innovation Management, Vol. 26, p. 551-565.

Bouillot C. (1999), " Mise en place de Progiciels de Gestion Intégrée à l'occasion de fusions et cessions d'entreprises dans un contexte international ", Systèmes d'information et management, Vol. 4, no 4, p. 91106.

Boutellier R., Gassmann O., Macho H. et Roux M. (1998), "Management of dispersed product development teams: the role of information technologies ", RED Management, Vol. 28, n¹, p. 13-26.

Brion S. (2005), " La coordination par la vigilance collective réciproque ", Revue française de gestion, Vol. 1, n¹54, p. 141157.

Brown S. L. et Eisenhardt K. M. (1995), " Product development: past research, present findings, and future directions ", $\mathrm{Aca}$ demy of Management Reviews, Vol. 20, $\mathrm{n}^{\circ} 2$, p.343-378.

Carlson JR et Zmud RW (1999), " Channel expansion theory and the experiential nature of media richness perceptions ", Academy of management journal, Vol. 42, n², p. $153-170$.

Chidambaram L. et Jones B. (1993), " Impact of Communication Medium and Computer Support on Group Perceptions and Performance: A Comparison of Face- toFace and Dispersed Meetings ", MIS Quarterly, Vol. 17, n4, p. 465-491.

Child J. et McGrath R.G. (2001), "Organizations unfettered: organizational form in an information intensive economy ", Academy of Management Journal, Vol. 44, p. 1135-1148.

Chin W. W et Dibbern J. (2010), " An introduction to a permutation based procedure for multi-group PLS analysis ", dans Handbook of Partial Least Squares: Concepts, methods and applications, Springer, Berlin Heidelberg, p. 171-193. 
Chin W., Marcolin B.L. et Newsted P.R. (2003), "A Partial Least Squares Latent Variable Modeling Approach for Measuring Interaction Effects: Results from a Monte Carlo Simulation Study and an ElectronicMail Emotion/Adoption Study ", Information Systems Research, Vol. 14, $\mathrm{n}^{\circ} 2$, p. 189-217.

Clark K. B., Chew W. B. et Fujimoto T. (1987), "Product Development in the World Auto Industry ", Brookings Papers on Economic Activity, Vol. 3, p. 729-781.

Clift T. B et Vandenbosch M. B. (1999), " Project Complexity and Efforts to Reduce Product Development Cycle Time ", Journal of Business Research, Vol. 45, n², p. 187-198.

Cramton C.D. (2001), " The mutual knowledge problem and its consequences for dispersed collaboration ", Organization Science, Vol. 12, n³, p. 346-371.

Cummings J.N., Espinosa J.A. et Pickering C.K. (2009), "Crossing Spatial and Temporal Boundaries in Globally Distributed Projects: A Relational Model of Coordination Delay ", Information Systems Research, Vol. 20, n³, p. 420-439.

Daassi M. et Favier M. (2007), " Le nouveau défi des équipes virtuelles : construire et maintenir une connaissance mutuelle ", Systèmes d'Information et Management, Vol. 12, n³, p. 3-30.

Daft R.L. et Lengel R. H. (1986), “Organizational Information Requirements, Media Richness and Structural Design ", Management Science, Vol. 32, n5, p. 554-571.

Desanctis et Monge (1999), "Introduction to the special issue: Communication processes for virtual organizations ", Organization Science, Vol. 10, n6, p. 693-703.

Durmusoglu S.S. et Barczak G. (2011), " The use of information technology tools in new product development phases: Analysis of effects on new product innovativeness, quality, and market performance ",
Industrial Marketing Management, Vol. 40, n², p. 321-330.

Dvir D., Lipovetsky S., Shenhar A. et Tishler A. (1998), "In search of project classification: a non-universal approach to project success factors ", Research Policy, Vol. 27, $n^{\circ} 9$, p. $915-935$.

Eisenhardt K.M. et Tabrizi B.N. (1995), “ Accelerating adaptive processes: Product innovation in the global computer industry ", Administrative Science Quarterly, Vol. 40, n 1 , p. 84-110.

Eppinger S.D. et Chitkara A.R. (2006), "The New Practice of Global ", MIT Sloan Management Review, Vol. 47, n4, p. 22-30.

Espinosa J.A., Cummings J.N., Wilson J.M. et Pearce B.M. (2003), "Team Boundary Issues Across Multiple Global Firms ", Journal of Management Information Systems, Vol. 19, n4, p. 157-190.

Esposito Vinzi V.E., Trinchera L. et Amato S. (2010), " PLS path modeling: from foundations to recent developments and open issues for model assessment and improvement ", dans Handbook of Partial Least Squares: Concepts, methods and applications, Springer, Berlin Heidelberg, p. 4782.

Fiol C.M. et O'Connor E.J. (2005), "Identification in face-to-face, hybrid, and pure virtual teams: Untangling the contradictions ", Organization Science, Vol. 16, n¹, p. 19-32.

Gassmann O. et von Zedtwitz M. (2003), “ Trends and determinants in managing virtual R\&D teams ", RED management, Vol. $33, n^{\circ} 3$, p. $243-262$.

Gerwin D. et Barrowman N. J. (2002), " An Evaluation of Research on Integrated Product Development ", Management Science, Vol. 48, n7, p. 938-953.

Gibson et Gibbs (2006), "Unpacking the Concept of Virtuality: The Effects of Geographic Dispersion, Electronic Dependen- 
ce, Dynamic Structure, and National Diversity on Team Innovation ", Administrative Science Quarterly, Vol. 51, p. 451-495.

Griffith T. L. et Neale M. A. (2001), "Information processing in traditional, hybrid, and virtual teams: From nascent knowledge to transactive memory ", Research in organizational behavior, Vol. 23, p. 379421.

Griffith T. L., Sawyer J. E. et Neale M. A. (2003), "Virtualness and knowledge in teams: Managing the love triangle of organizations, individuals, and information technology ", MIS Quarterly, Vol. 27, n²2, p. 265-287.

Grinter R.E., Herbsleb J. D. et Perry D. E. (1990), "The Geography of Coordination: Dealing with Distance in R\&D Work ", ACM SIGGROUP conference Phoenix, Arizona, United States.

Gupta A., Mattarelli E., Seshasai S. et Broschak J. (2009), "Use of collaborative technologies and knowledge sharing in co-located and distributed teams: Towards the 24-h knowledge factory ", The Journal of Strategic Information Systems, Vol. 18, n³, p. 147-161.

Hair J.F. Sarstedt M., Ringle C.M. et Mena J.A. (2011), "An assessment of the use of partial least squares structural equation modeling in marketing research ", Journal of the Academy of Marketing Science, Vol. 40, n³, p. 1-20.

Hameri A.P. et Nihtilä J. (1997), " Distributed New Product Development Project Based on Internet and World-Wide Web: A Case Study ", Journal of product innovation management, Vol. 14, n², p. 77-87.

Harvey M.G. et Griffith D.A. (2007), "The role of globalization, time acceleration, and virtual global teams in fostering successful global product launches ", Journal of Product Innovation Management, Vol. 24, n5, p. 486-501.
Hertel G., Geister S. et Konradt U. (2005), “ Managing virtual teams: A review of current empirical research ", Human Resource Management Review, Vol. 15, p.69-95.

Hertel G., Konradt U. et Orlikowski B. (2004), "Managing distance by interdependence: Goal setting, task interdependence, and team-based rewards in virtual teams ", European Journal of Work and Organizational Psychology, Vol. 13, n¹, p. 1-28.

Hoegl M. et Gemuenden H. G. (2001), " Teamwork Quality and the Success of Innovative Projects: A Theoretical Concept and Empirical Evidence ", Organization Science, Vol. 12, n4, p. 435-449.

Hoegl M., Ernst H. et Proserpio L. (2007), “ How Teamwork Matters More as Team Member Dispersion Increases ", Journal of Product Innovation Management, Vol. 24, n², p. 156-165.

Hoegl M., Weinkauf K. et Gemuenden H.G. (2004), "Interteam Coordination, Project Commitment, and Teamwork in Multiteam R\&D Projects: A Longitudinal Study ", Organization Science, Vol. 15, $\mathrm{n}^{\circ} 1$, p. 38-55.

Holland S., Gaston K. et Gomes J. (2000), "Critical success factors for cross-functional teamwork in new product development ", International Journal of Management Reviews, Vol. 2, n³, p. 231-259.

Hoopes D.G. et Postrel S. (1999), "Shared knowledge, "glitches," and product development performance ", Strategic Management Journal, Vol. 20, n9, p. 837-865.

Howells J. R. (1995), " Going global: The use of ICT networks in research and development ", Research Policy, Vol. 24, n², p. 169-184.

Kahn K. B. et McDonough E. F. (1997), "An Empirical Study of the Relationships among Colocation, Integration, Performance, and Satisfaction ", Journal of Pro- 
duct Innovation Management, Vol. 14, n³, p. 161-178.

Kanawattanachai P. et Yoo Y. (2007), "The Impact of Knowledge Coordination on Virtual Team Performance over Time ", MIS Quarterly, Vol. 31, n4, p. 783-808.

Kiesler S. et Cummings J. N. (2002), "What Do We Know about Proximity and Distance in Work Groups? ", dans Distributed work, Cambridge and London: MIT Press.

Kim B. et Kim J. (2009), "Structural factors of NPD (new product development) team for manufacturability ", International Journal of Project Management, Vol. 27, p. 690-702.

Kirkman B. L. et Mathieu J. E. (2005), "The Dimensions and Antecedents of Team Virtuality ", Journal of Management, Vol. 31, n5, p. 700-718.

Kotlarsky J., Van Fenema P.C. et Willcocks L.P. (2008), "Developing a knowledgebased perspective on coordination: The case of global software projects ", Information \& Management, Vol. 45, $\mathrm{n}^{\circ} 2$, p. 96-108.

Koufteros X. A., Vonderembse M. A et Doll W. J. (2002), " Integrated product development practices and competitive capabilities: the effects of uncertainty, equivocality, and platform strategy ", Journal of Operations Management, Vol. 20, n4, p. 331-355.

Krishnan V. et Ulrich K. T. (2001), "Product development decisions: A review of the literature ", Management Science, Vol. 47, $\mathrm{n}^{\circ} 1$, p. 1-21.

Kumar P. P. (2005), "Effective Use of Gantt Chart for Managing Large Scale Projects ", Cost Engineering, Vol. $47, n^{\circ} 7$, p. 14-21.

Langevin P. (2002), " Efficacité et contrôle des équipes virtuelles : une revue ", Comptabilité- Contrôle-Audit, Vol. 2, p. 87-107.
Lee A. S. (1994), "Electronic Mail as a Medium for Rich Communication: An Empirical Investigation Using Hermeneutic Interpretation ", MIS Quarterly, Vol. 18, n², p. $143-157$.

Leenders M. et Wierenga B. (2002), "The effectiveness of different mechanisms for integrating marketing and R\&D ", Journal of Product Innovation Management, Vol. 19, n4, p. 305-317.

Levina N. et Vaast E. (2006), "Turning a community into a market: A practice perspective on information technology use in boundary spanning ", Journal of Management Information Systems, Vol. 22, n4, p. 13-37.

Malhotra A., Majchrzak A., Carman R. et Lott V. (2001), "Radical Innovation without Collocation: A Case Study at Boeing-Rocketdyne ", MIS Quarterly, Vol. 25, n², p. 229-249.

Malone T. W. et Crowston K. (1990), "What is Coordination Theory and How Can It Help Design Cooperative Work Systems? ", ACM conference, Los Angeles.

Marciniak R. et F. Rowe (1999), "Styles de coordination avec les sous-traitants, expérience commune et performance économique : le cas de trois projets dans le bâtiment ", Systèmes d'Information et Management, Vol. 4, n², p. 37-64.

Marcoulides G. A. et Saunders C. (2006), “ PLS: A Silver Bullet? ", MIS Quarterly, Vol. 30, n², p. 3-9.

McDonough E. F., Kahn K. B. et Barczak G. (2001), "An investigation of the use of global, virtual, and colocated new product development teams ", Journal of Product Innovation Management, Vol. 18, $\mathrm{n}^{\circ} 2$, p. $110-120$.

Merminod V., Mothe C. et Rowe F. (2009), "Effets de Product Lifecycle Management sur la fiabilité et la productivité : une comparaison entre deux contextes de déve- 
loppement produit,, M@n@gement, Vol. 12, n4, p. 294-331.

Merminod V. et Rowe F. (2012), "How does PLM technology support knowledge transfer and translation in new product development? Transparency and boundary spanners in an international context", Information and Organization, Vol. 22, n4, p. 295-322.

Michaux V. (2005), "Performance des processus de coordination à distance : une approche exploratoire ", Systèmes d'Information et Management, Vol. 12, $\mathrm{n}^{\circ} 1$, p. 11-39.

Mihm J., Loch C. et Huchzermeier A. (2003), "Problem-Solving Oscillations in Complex Engineering Projects ", Management Science, Vol. 49, n6, p. 733-750.

Moenaert R. K., Caeldries F., Lievens A. et Wauters E. (2000), " Communication flows in international product innovation teams ", Journal of Product Innovation Management, Vol. 17, n. 5, p. 360-377.

Nambisan S. (2003), "Information Systems as a Reference Discipline for New Product Development ", MIS Quarterly, Vol. 27, n¹, p. 1-18.

Nardi B. et Whittaker S. (2002), "The Place of Face-to-Face Communication in Distributed Work ", dans Distributed Work. MIT Press, MA, Cambridge, p. 83-110.

O'Leary M.B. et Cummings J.N. (2007), "The Spatial, Temporal, and Configurational Characteristics of Geographic Dispersion in Teams ", MIS Quarterly, Vol. 31, n³, p. 433-452.

Olson E. M., Walker O. C., Ruekert R. W. et Bonner J. M. (2001), "Patterns of cooperation during new product development among marketing, operations and R\&D: Implications for project performance ", The Journal of Product Innovation Management, Vol. 18, p. 258-271.
Orlikowski (2002), "Knowing in Practice: Enacting a Collective Capability in Distributed Organizing ", Organization Science, Vol. 13, n4, p. 249-273.

Oshri I., Kotlarsky J. et Willcocks L.P. (2007), " Global software development: Exploring socialization and face-to-face meetings in distributed strategic projects ", The Journal of Strategic Information Systems, Vol. 16, n' 1 , p. 25-49.

Ozer M. (2000), "Information Technology and New Product Development: Opportunities and Pitfalls ", Industrial Marketing Management, Vol. 29, n5, p. 387-396.

Patrashkova R. R. et McComb S. A. (2004), "Exploring why more communication is not better: insights from a computational model of cross-functional teams ", Journal of Engineering and Technology Management, Vol. 21, n¹-2, p. 83-114.

Pauleen D.J. et Yoong P. (2001), "Relationship building and the use of ICT in boundary-crossing virtual teams: a facilitator's perspective ", Journal of Information Technology, Vol. 16, p. 205-220.

Pavlou P.A. et El Sawy, O. A. (2010), " The Third Hand: IT-Enabled Competitive Advantage in Turbulence through Improvisational Capabilities ", Information Systems Research, Vol. 21, n³, p. 1-41.

Pavlou P.A. et El Sawy O.A. (2006), " From IT leveraging competence to competitive advantage in turbulent environments: The case of new product development ", Information Systems Research, Vol. 17, n³, p. $198-227$.

Pearce J. L. et Gregersen H. B. (1991), " Task interdependence and extrarole behavior: A test of the mediating effects of felt responsibility ", Journal of Applied Psychology, Vol. 76, n6, p. 838-844.

Pinto M. B. et Pinto J. K. (1990), "Project Team Communication and Cross Functional Cooperation in New Program Deve- 
lopment ", Journal of Product Innovation Management, Vol. 7, n³, p. 200-212.

Pinto M. B., Pinto J. K., et Prescott J. E. (1993), "Antecedents and Consequences of Project Team Cross-functional Cooperation. ", Management Science, Vol. 39, n¹0, p. 1281-1297.

Podsakoff P. M. et Organ D.W. (1986), “ SelfReports in Organizational Research: Problems and Prospects ", Journal of Management, Vol. 12, n4, p. 531.

Rönkkö M. et Ylitalo J. (2011), “ PLS marker variable approach to diagnosing and controlling for method variance ", ICIS.

Salomo S., Keinschmidt E.J. et De Brentani U. (2010), " Managing New Product Development Teams in a Globally Dispersed NPD Program ", Journal of Product Innovation Management, Vol. 27, $\mathrm{n}^{\circ} 7$, p. 955971.

Salter A. et Gann D. (2003), "Sources of ideas for innovation in engineering design ", Research Policy, Vol. 32, n8, p. 1309-1324.

Sanchez R. et Mahoney J.T. (1996), " Modularity, flexibility, and knowledge management in product and organization design ", Strategic Management Journal, Special Issue, Vol. 17, p. 63-76.

Schultze U. et Orlikowski W. J. (2001), " Metaphors of virtuality: shaping an emergent reality ", Information and Organization, Vol. 11, n¹, p. 45-77.

Sole D. et Edmondson A. (2002), "Situated Knowledge and Learning in Dispersed Teams ", British Journal of Management, Vol. 13, n², p. 17-34.

Sosa M.E., Eppinger S.D., Pich M., McKendrick D.G., Suzanne K. et Stout S.K. (2002), "Factors That Influence Technical Communication in Distributed Product Development: An Empirical Study in the Telecommunications Industry", IEEE
Transactions on Engineering Management, Vol. 49, n²1, p. 45-58.

Sosa M.E., Eppinger S.D. et Rowles C.M. (2004), "The Misalignment of Product Architecture and Organizational Structure in Complex Product Development ", Management Science, Vol. 50, $\mathrm{n}^{\circ} 12$, p. 16741689.

Sosik J. J., Kahai S.S. et Piovoso M.J. (2009), "Silver Bullet or Voodoo Statistics?", Group \& Organization Management, Vol. $34, n^{\circ} 1$, p. $5-36$.

Srivastava A., Bartol K. M. et Locke E. A. (2006), " Empowering leadership in management teams: Effects on knowledge sharing, efficacy, and performance ", The Academy of Management Journal, Vol. 49, no6, p. 1239-1251.

Stone N. J. et Posey M. (2008), “ Understanding coordination in computer-mediated versus face-to-face groups ", Computers in Human Behavior, Vol. 24, n³, p. 827851.

Subrahmanian E., Monarch I., Konda S., Granger H., Milliken R. et Westbergerg A. (2003), "Boundary objects and prototypes at the interfaces of engineering design ", Computer Supported Cooperative Work (CSCW), Vol. 12, n², p. 185-203.

Sun H. (2000), "Current and future patterns of using advanced manufacturing technologies ", Technovation, Vol. 20, n¹1, 631641.

Tan C. L et Vonderembse M. A. (2006), " Mediating effects of computer-aided design usage: From concurrent engineering to product development performance ", Journal of Operations Management, Vol. 24, n5, p. 494-510.

Taylor S. et Spicer A. (2007), "Time for space: A narrative review of research on organizational spaces ", International Journal of Management Reviews, Vol. 9, $\mathrm{n}^{\circ} 4$, p. 325-346. 
Tenenhaus M., Esposito Vinzi V., Chatelin Y. et Lauro C. (2005), " PLS path modeling ", Computational Statistics \& Data Analysis, Vol. 48, n¹, p. 159-205.

Thompson J. D., (1967), Organizations in action, McGraw-Hill, New York.

Tidd J. et Bodley K. (2002), " The influence of project novelty on the new product development process ", RED Management, Vol. 32, n², p. 127-138.

Warkentin M.E., Sayeed L. et Hightower R. (1997), "Virtual Teams versus Face-toFace Teams: An Exploratory Study of a Web-based Conference System ", Decision Sciences, Vol. 28, n4, p. 975-996.

Watson-Manheim M.B., Chudoba K.M. et Crowston K. (2011), " Perceived discontinuities and constructed continuities in vir- tual work ", Information Systems Journal, Vol. 22, n¹, p. 29-52.

Wetzels M., Odekerken-Schröder G. et Van Oppen C. (2009), " Using PLS path modeling for assessing hierarchical construct models: Guidelines and empirical illustration ", MIS Quarterly, Vol. 33, n¹, p. 177195.

Yakura E. K. (2002), "Charting Time: Timelines as Temporal Boundary Objects ", Academy of Management Journal, Vol. 45, n5, p. 956-970.

Yalcin A., Khemuka A. et Deshpande P. (2005), "Modelling inter-task dependencies and control of workflow managements systems based on supervisory control theory ", International Journal of Production Research, Vol. 43, n²0, p. 4359-4379.

\section{ANNEXES}

Annexe A « Caractéristiques des projets de NPD "

\begin{tabular}{|c|c|c|c|c|}
\hline Projets & Industries & Tailles des équipes & $\begin{array}{c}\text { Interdépendance } \\
\text { des tâches }\end{array}$ & Degré d'innovation \\
\hline 1 & Aérospatiale & 33 & 3,91 & 4,24 \\
\hline 2 & Microélectronique & 35 & 3,45 & 4,1 \\
\hline 3 & Télécommunication & 20 & 3,56 & 3,37 \\
\hline 4 & Équipement industriel & 21 & 3,37 & 3,82 \\
\hline 5 & Équipement industriel & 24 & 3,62 & 4,58 \\
\hline 6 & Équipement automobile & 38 & 3,5 & 3,94 \\
\hline 7 & Mécanique & 38 & 3,71 & 3,76 \\
\hline Moyenne & & 29,86 & 3,59 & 3,97 \\
\hline Écart-type & & 7,95 & 0,18 & 0,39 \\
\hline
\end{tabular}


Annexe B " Instruments de mesure "

\begin{tabular}{|c|c|c|}
\hline Variable & Indicateurs réflexifs & Codes \\
\hline \multirow{7}{*}{$\begin{array}{c}\text { Efficacité } \\
\text { de la coordination } \\
\text { des tâches } \\
\text { Leenders et Wierenga } \\
\text { (2002) }(\alpha: 0,91) \\
\text { Hoegl et Gemuenden } \\
\quad \text { (2001) ; Hoopes } \\
\text { et Postrel (1999) }\end{array}$} & $\begin{array}{l}\text { La communication des informations s'est déroulée sans entrave entre les } \\
\text { membres de l'équipe projet }\end{array}$ & COORD_1 \\
\hline & $\begin{array}{l}\text { Les membres de l'équipe projet se sont davantage perçus comme des co- } \\
\text { équipiers que comme des rivaux }\end{array}$ & COORD_2 \\
\hline & $\begin{array}{l}\text { Lors des désaccords pendant le projet, les membres de l'équipe projet } \\
\text { ont été capables de les résoudre }\end{array}$ & COORD_3 \\
\hline & $\begin{array}{l}\text { Les membres de l'équipe projet se sont entraidés pour exécuter plus effi- } \\
\text { cacement leurs tâches }\end{array}$ & COORD_4 \\
\hline & \begin{tabular}{|l|} 
Les membres de l'équipe projet ont omis de se communiquer des infor- \\
mations importantes pendant le projet $(\mathrm{R})^{7}$
\end{tabular} & R_COORD_5 \\
\hline & $\begin{array}{l}\text { Les membres de l'équipe projet se sont reproché des erreurs entre eux } \\
\text { pendant le projet (R) }\end{array}$ & R_COORD_6 \\
\hline & $\begin{array}{l}\text { Vous avez été obligé de supposer ce que faisaient d'autres membres de } \\
\text { l'équipe projet par manque de connaissances et d'informations }(\mathrm{R})\end{array}$ & R_COORD_9 \\
\hline $\begin{array}{l}\text { Communications } \\
\text { en présentiel } \\
\text { Hertel et al. } 2004 ; \\
\text { Kirkman et al. }(2004)\end{array}$ & $\begin{array}{l}\text { Quelle a été la fréquence des échanges en présentiel entre les membres } \\
\text { de l'équipe projet? }\end{array}$ & COM_PRES \\
\hline $\begin{array}{c}\text { TIC communications } \\
\text { synchrones } \\
\text { Chidambaram et Jones } \\
\text { (1993) ; Boutellier et al. } \\
\text { (1998) ; Durmusoglu et } \\
\text { Barczak (2011) }\end{array}$ & Votre usage de la visioconférence a été... & TIC_1_SYNC \\
\hline $\begin{array}{c}\text { TIC d'aide } \\
\text { à la conception } \\
\text { Pavlou et El Sawy (2006) }\end{array}$ & $\begin{array}{l}\text { Votre usage des technologies pour concevoir et développer le produit } \\
\text { (outils de prototypage virtuel, CAD (Computer Aided Design) ; CAM } \\
\text { (Computer Aided Manufacturing) a été... }\end{array}$ & TIC_4_CONC \\
\hline \multirow{3}{*}{$\begin{array}{l}\text { TIC pour organiser } \\
\text { les tâches } \\
\text { Pavlou et El Sawy (2006) }\end{array}$} & $\begin{array}{l}\text { Votre usage de documents (rapports d'avancement, données, plans, etc.) } \\
\text { a été... }\end{array}$ & TIC_5_ORGA \\
\hline & $\begin{array}{l}\text { Votre usage des technologies pour organiser les tâches, les jalons et af- } \\
\text { fecter les ressources du projet (MS-Project) a été... }\end{array}$ & TIC_6_ORGA \\
\hline & $\begin{array}{l}\text { Votre usage des technologies pour organiser, partager, compiler, valoriser } \\
\text { les informations, les connaissances et les bonnes pratiques (bases de } \\
\text { données Access/Excel, espaces de stockage partagés, etc.) a été... }\end{array}$ & TIC_7_ORGA \\
\hline \multirow[b]{2}{*}{$\begin{array}{l}\text { Niveau de distribution } \\
\text { des équipes projets } \\
\text { O'Leary et Cummings } \\
\text { (2007) }\end{array}$} & $\sum_{i-j}^{k} \frac{\left(k m_{i-j}{ }^{*} n_{i}{ }^{*} n_{j}\right)}{\frac{\left(N^{2}-N\right)}{2}}$ & DIST_SPA_O \\
\hline & $\begin{array}{l}\text { Distance temporelle } \sum_{i-j}^{k} \frac{\left(\text { fuseaux }- \text { horaires }_{i-j} * n_{i}{ }^{*} n_{j}\right)}{\frac{\left(N^{2}-N\right)}{2}} \\
k m_{i-j}=\text { nombre de kilomètres qui séparent les sites " } \mathrm{i} \text { " et " } \mathrm{j} \text { " } \\
\mathrm{n}_{i}=\text { taille de l'équipe au sein du site " } \mathrm{i} \text { " } \\
n_{j}=\text { taille de l'équipe au sein du site " } \mathrm{j} \text { " } \\
\mathrm{N}=\text { taille totale de l'équipe }\end{array}$ & DIST_TEMP_O \\
\hline Appartenance au projet & Numéro du projet auquel le répondant appartient & PROJET \\
\hline \multirow{2}{*}{$\begin{array}{c}\text { Interdépendance } \\
\text { des tâches } \\
\text { Pearce et Gregersen (1991) } \\
(\alpha: 0,76)\end{array}$} & $\begin{array}{l}\text { Les membres de l'équipe projet ont accompli leurs tâches indépendam- } \\
\text { ment des autres membres de l'équipe (R) }\end{array}$ & R_INTER_TA_1 \\
\hline & \begin{tabular}{|l|} 
Les membres de l'équipe projet dépendaient les uns des autres pour obte- \\
nir les informations et le matériel nécessaires pour accomplir leurs tâches
\end{tabular} & INTER_TA_2 \\
\hline
\end{tabular}

(R) Reverse coding (codage inversé). 
Annexe C « Niveaux de distribution des équipes projets "

\begin{tabular}{|c|c|c|c|}
\hline Projets & $\begin{array}{c}\text { Indices de distance } \\
\text { spatiale }\end{array}$ & $\begin{array}{c}\text { Indices de distance } \\
\text { temporelle }\end{array}$ & Nombre de sites \\
\hline 1 & 407 & 0 & 6 \\
\hline 2 & 1883 & 1,43 & 5 \\
\hline 3 & 5296 & 4,63 & 5 \\
\hline 4 & 2408 & 1,94 & 4 \\
\hline 5 & 128 & 0 & 3 \\
\hline 6 & 829 & 0,49 & 3 \\
\hline 7 & 5124 & 1,97 & 4 \\
\hline Minimum & $\mathbf{1 2 8}$ & $\mathbf{0}$ & $\mathbf{3}$ \\
\hline Maximum & $\mathbf{5 2 9 6}$ & $\mathbf{4 . 6 3}$ & $\mathbf{4 , 2 9}$ \\
\hline Moyennes & $\mathbf{2 2 9 6 , 4 3}$ & $\mathbf{1 , 4 9}$ & $\mathbf{1 , 0 3}$ \\
\hline Ecarts-types & $\mathbf{1 9 8 5 , 7 1}$ & $\mathbf{1 , 5 0}$ & \\
\hline
\end{tabular}

Annexe D «Corrélations entre les indicateurs des mesures »

\begin{tabular}{|c|c|c|c|c|c|c|c|c|}
\hline & $\begin{array}{c}\text { Efficacité } \\
\text { Coord. }\end{array}$ & $\begin{array}{c}\text { Com. } \\
\text { Présentiel }\end{array}$ & Interdép. & Distrib. & TIC Conc. & TIC Orga. & $\begin{array}{c}\text { TIC } \\
\text { Com. Sync. }\end{array}$ & Projet \\
\hline DIST_SPA & $-0,2404$ & $-0,3671$ & $-0,0277$ & 0,9652 & $-0,0408$ & $-0,0498$ & 0,1445 & 0,0067 \\
\hline DIST_TEMP & $-0,2609$ & $-0,493$ & $-0,1137$ & 0,9705 & 0,0591 & $-0,1106$ & $-0,037$ & $-0,0683$ \\
\hline COM_PRES & 0,3251 & 1 & 0,1199 & $-0,4469$ & 0,0416 & 0,3097 & 0,0338 & $-0,0142$ \\
\hline INTER_TA_1_R & 0,2239 & 0,1272 & 0,9129 & $-0,0811$ & 0,203 & 0,0551 & 0,1644 & $-0,1163$ \\
\hline INTER_TA_2 & 0,1232 & 0,048 & 0,6706 & $-0,027$ & 0,1666 & 0,1797 & $-0,099$ & 0,0861 \\
\hline COORD_1 & 0,8031 & 0,1395 & 0,125 & $-0,0818$ & 0,2373 & 0,3174 & $-0,047$ & $-0,1515$ \\
\hline COORD_2 & 0,7257 & 0,2373 & 0,1955 & $-0,1278$ & 0,1887 & 0,3342 & 0,0802 & 0,0604 \\
\hline COORD_3 & 0,813 & 0,3784 & 0,1086 & $-0,4113$ & 0,1627 & 0,2559 & $-0,0566$ & 0,0239 \\
\hline COORD_4 & 0,7723 & 0,3285 & 0,1506 & $-0,2044$ & 0,1814 & 0,2379 & $-0,1418$ & $-0,1879$ \\
\hline COORD_5_R & 0,6373 & 0,1053 & 0,242 & $-0,2432$ & 0,2783 & 0,0696 & $-0,2528$ & $-0,3217$ \\
\hline COORD_6_R & 0,6132 & 0,1699 & 0,1891 & $-0,034$ & 0,039 & 0,1645 & $-0,1321$ & $-0,004$ \\
\hline COORD_9_R & 0,6405 & 0,2773 & 0,1868 & $-0,1462$ & $-0,0513$ & 0,2586 & $-0,0746$ & $-0,0297$ \\
\hline PROJET & $-0,1234$ & $-0,0142$ & $-0,0542$ & $-0,0337$ & $-0,2841$ & $-0,1086$ & 0,1171 & 1 \\
\hline TIC_1_SYNC & $-0,1186$ & 0,0338 & 0,0858 & 0,0517 & $-0,1272$ & 0,1739 & 1 & 0,1171 \\
\hline TIC_4_CONC & 0,2251 & 0,0416 & 0,2299 & 0,0115 & 1 & 0,2312 & $-0,1272$ & $-0,1195$ \\
\hline TIC_5_ORGA & 0,3213 & 0,3282 & 0,2137 & $-0,1932$ & 0,2713 & 0,8666 & 0,1763 & $-0,2841$ \\
\hline TIC_6_ORGA & 0,0913 & 0,2066 & 0,0803 & 0,0833 & 0,1647 & 0,5869 & 0,141 & $-0,1745$ \\
\hline TIC_7_ORGA & 0,2724 & 0,1844 & $-0,0305$ & 0,026 & 0,1031 & 0,8503 & 0,1047 & $-0,0246$ \\
\hline
\end{tabular}

